\title{
Aktueller Stand der anatomischen Schulterendoprothetik
}

\author{
Petra Magosch, Sven Lichtenberg, Peter Habermeyer
}

\section{Zusammenfassung}

Die moderne Ära der Schulterendoprothetik resultiert aus der Weiterentwicklung des 1951 von Charles Neer entwickelten Neer-Prothesensystems. Dieser Artikel gibt einen Überblick über die Entwicklung und die Implantatcharakteristika der Schulterendoprothesen mit ihren Vor- und Nachteilen sowie den Indikationen zur anatomischen Rekonstruktion des Glenohumeralgelenks. Abschließend werden die Langzeitergebnisse in Abhängigkeit der Ätiologie der Omarthrose dargestellt.

\section{Current Status of Anatomic Shoulder Arthroplasty}

The modern era of shoulder arthroplasty has resulted from the further development of the Neer prosthesis system first described by Charles Neer in 1951. The present article provides an overview of the development and implant characteristics of shoulder arthroplasties with their advantages and disadvantages as well as indications for an anatomic reconstruction of the glenohumeral joint. Finally, long-term results in dependency on the aetiology of the osteoarthritis are presented

\section{Einleitung}

Die Schulterendoprothetik umspannt ein weitreichendes Indikationsspektrum von den degenerativen, entzündlichen über infektiöse, tumoröse, instabilitätsassoziierte und neurogene bis hin $\mathrm{zu}$ den traumatischen und posttraumatischen Gelenkflächendefekten. Jede dieser Erkrankungs- und Traumaformen erfordert ein eigenes Regime und entsprechendes Implantat. Ziel der modernen Schulterendoprothetik ist die anatomische und damit auch biomechanische Rekonstruktion des Glenohumeralgelenks. Bei Rotatorenmanschetteninsuffizienz und nicht rekonstruierbarer Rotatorenmanschette unterschiedlicher Ätiologien bleibt unter Berücksichtigung des Patientenalters die Implantation einer inversen, extraanatomischen Schulterprothese, welche jedoch nicht Gegenstand dieses Artikels ist.

OP-JOURNAL 2013; 29: 82-97

(c) Georg Thieme Verlag KG Stuttgart · New York DOI http://dx.doi.org/10.1055/s-0032-1328421
Entwicklung und aktueller Stand des

\section{Standardschaftprothesen}

Die moderne Ära der Schulterprothetik begann Anfang der 70er-Jahre des 20. Jahrhunderts mit der Verbesserung des seit 1951 vorliegenden „Neer“-Prothesensystems (Neer 1974), das ursprünglich als Frakturprothese zur Versorgung von Kopftrümmerbrüchen entwickelt wurde. Das darauf weiterentwickelte Implantat (Neer-II-System) ermöglichte eine erste individuelle Anpassung durch unterschiedliche Schaftlängen und -dicken sowie unterschiedliche Kopfgrößen des integrierten Prothesenkopfs. Aber mit nur einem Kopfradius von $44 \mathrm{~mm}$ und nur 2 verschiedenen Kalottenhöhen von 15 und $22 \mathrm{~mm}$ konnte es weder in der Koronar- noch in der Axialebene die Geometrie des proximalen Humerus für jeden Fall identisch nachbilden [1]. Da sich die Größe des Humeruskopfs aus einem konstanten Längenverhältnis von Kopfradius und Kalottenhöhe in der Koronarebene definiert [2], ist mit der Standard-Neer-Prothese keine komplette Replikation der individuellen Kalottenanatomie zu erreichen [3].

Die 2. Generation der Schulterprothesen, welche weiterhin auf der Neer-II-KopfSchaft-Geometrie basiert, bietet die Möglichkeit der Auswahl von verschieden dimensionierten modularen Kopfund Schaftgrößen. Das modulare Konzept bietet neben dem Vorteil operationstechnischer Vereinfachung bei Implantation und Revision die Möglichkeit einer der Weichteilsituation angepassten Kalottenauswahl unter besonderer Berücksichtigung des lateralen humeralen Offsets [4]. Durch Änderung der korrespondierenden Radien von Kopf und Glenoid (engl. mismatch) wurde ein Roll-Gleit-Mechanismus freigegeben.

In einer Vergleichsstudie der 4 häufigsten in den USA angewandten Schulterprothesen der 2. Generation (Biomet, DePuy, Intermedics, Kirschner; Abb. 1) zeigte sich, dass keine der Prothesen eine identische Replikation der Kopfgeometrie, insbesondere des Rotationszentrums ermöglichte.

Neuere Modifikationen der Prothesen der 2. Generation erlauben auch eine exzentrische Positionierung der Kopfkalotte (Global Shoulder, DePuy; Bio-Modular, Biomet; HAS, Howmedica). Eine Möglichkeit zur variablen Einstellung des Inklinationswinkels besteht weiterhin nicht. Somit wird mit diesen Implantaten eine anatomische Anpassung der Kalotte auf der humeralen Resektionsfläche zwangsläufig nicht immer erreicht.

Die 1991 von Walch und Boileau [5] konzeptionierte 3. Generation (Aequalis, Tornier; Abb. 2) verfolgt mit einem 3fach modularen System das Ziel, die Prothese der Kopfanatomie anzupassen und nicht durch Resektion den proximalen Humerus der Prothese anzugleichen. Somit ist der anatomische Hals die wesentliche und kritische Landmarke, die allein als Resektionslinie die Richtung der 


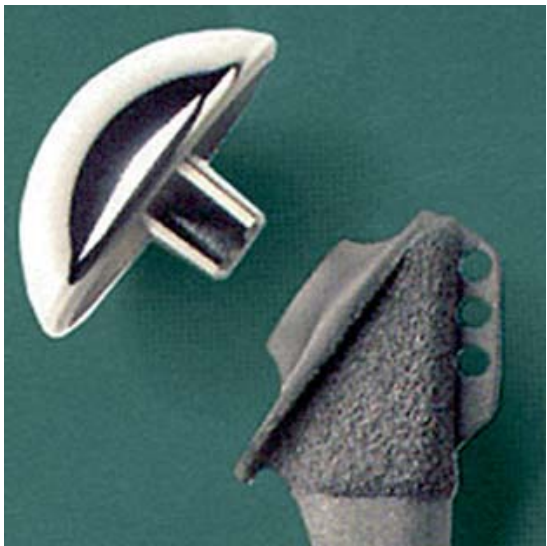

Abb. 1 Humeruskopfprothese der zweiten Generation. Bio-Modular-Prothese (Fa. Biomet Deutschland $\mathrm{GmbH}$ ).

Kopfresektion bestimmt. Da der zirkulär darstellbare anatomische Hals die Inklination und Retrotorsion determiniert, kann bei Resektion planar zum anatomischen Hals der individuellen Anatomie jedes einzelnen Patienten Rechnung getragen werden. Den individuellen Inklinationswinkel rekonstruiert man mit einem modularen Winkeladapter in Schritten von jeweils $5^{\circ}$ zwischen 125 und $140^{\circ}$. Eine variable Einstellung der Inklination ist auch deswegen sinnvoll, weil es dem Operateur nicht in jedem Fall gelingt, den von der Prothese vorgegebenen Inklinationswinkel einzuhalten, was zu einem Mismatch zwischen Resektionsfläche und Prothesenrückfläche führt. Durch die Möglichkeit, die Kopfkalotte exzentrisch auf dem Winkeladapter zu verankern, kann man das nach medial und dorsal versetzte Drehzentrum prothetisch rekonstruieren [6, 7]. Die Wiederherstellung des Originalrotationszentrums gewährleistet eine anatomische Kinematik, verhindert eine erhöhte Spannung an der Rotatorenmanschette und vermindert eine exzentrische Pfannenüberlastung [8].

Die 4. Generation der Schulterprothesen ermöglicht eine stufenfreie Modularität um 3 Achsen im Sinne einer dreidimensionalen Variabilität. Sowohl von Gerber (Anatomica, Fa. Zimmer) als auch von Habermeyer (Univers, Fa. Arthrex; Abb. 3) wurden 1997 Systeme vorgestellt, die neben einer variablen Adjustierung des Inklinationswinkels und der Kopfexzentrizität die starre Verbindung zwischen Prothesenschaft und Kalotte mit einer Versionsmöglichkeit im KopfHals-Bereich freigaben. Dadurch wurde die Kopfgeometrie von der Schaftposition unabhängig und ermöglichte eine

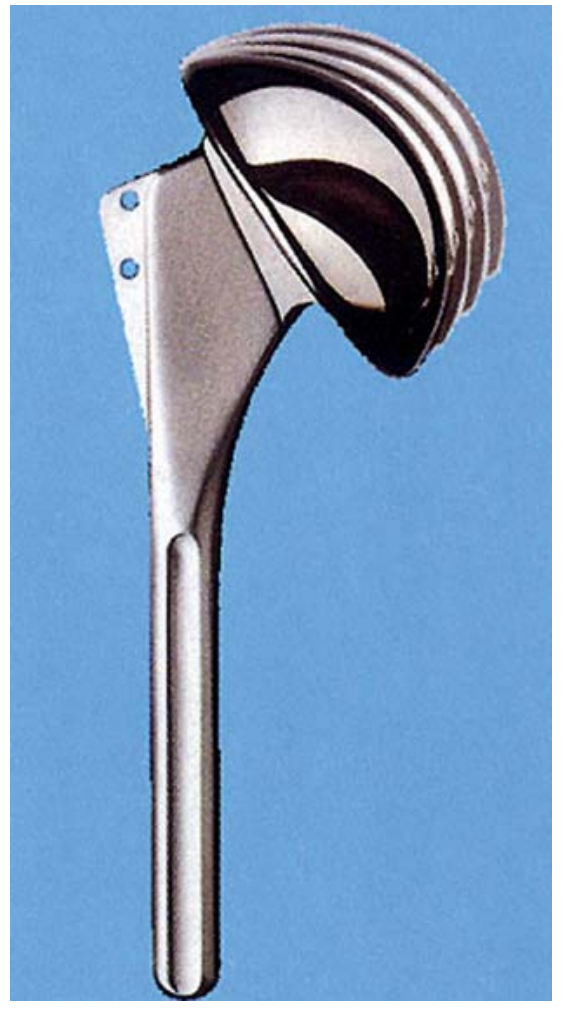

Abb. 2 Humeruskopfprothese der dritten Generation. Aequalis Prothese (Fa. Tornier).

exaktere Kalottenrekonstruktion. Verstellungen der Kalotte sind damit in 3 Ebenen - um die Schaftachse (Ante-, Retroversion), in der Koronarebene (Inklinationswinkel) und in der Sagittalebene (posteromedialer Offset) - möglich. Die freie Verstellbarkeit zwischen Schaft und Kalotte erlaubt es, dass eine Torsionsänderung im Sinne einer Ante- und Retrotorsion um die Kopf-Hals-Achse möglich wird. Dies ist deswegen von Bedeutung, da der Prothesenschaft der Richtung des Markraumkanals folgt und somit die Position des Prothesenkopfs determiniert $[1,9,10]$.

Um im Fall eines Wechsels auf inverse Implantate den dafür notwendigen Schaftwechsel zu vermeiden, bieten die neuen Schaftprothesen der 4. Generation die Möglichkeit, den modularen Kopfteil gegen eine inverse Humeruspfanne zu tauschen (Fa. Lima, Fa. Smith \& Nephew, Fa. Zimmer). Auch an der Pfannenseite können bei Verwendung von Metalback-Systemen im Fall des Wechsels die Polyethylen-Inlays gegen Glenosphären ausgetauscht werden (Fa. Arthrex, Fa. Lima, Fa. Mathys, Fa. Zimmer).
Schaftfreie (metaphysär verankerte) Prothesen

Besonders für jüngere Patienten mit degenerativen Veränderungen des Schultergelenks muss trotz verbesserter Standzeiten der klassischen Endoprothesen mit Wechseloperationen im weiteren Verlauf des Lebens gerechnet werden. Klassische Schaftprothesen erfordern im Revisionsfall Schaftosteotomien mit erheblichen Morbiditätsraten.

Herkömmliche Cup-Prothesen sind ebenfalls keine Ideallösung, da sie einen primären oder sekundären Pfanneneinbau extrem erschweren.

Die modernste Lösung dieses Problems stellt der seit 2005 erhältliche schaftfreie Humeruskopfersatz (Abb.4; Eclipse, Fa. Arthrex; T.E.S.S., Fa. Biomet;, Affinis short, Fa. Mathys; Simplicity, Fa. Tornier) als goldener Mittelweg dar. Der schaftfreie Humeruskopfersatz bietet folgende Vorteile:

- anatomische Kopfgrößen in 2-mmSchritten

- ideale Anpassung des humeralen Offset durch Ausrichtung auf die Kortikalisränder am resezierten anatomischen Hals

- Kalottenpositionierung unabhängig von der Schaftachse, insbesondere bei der posttraumatischen Arthrose

- freier Zugang zum Glenoid bei der Pfannenimplantation (auch bei der Konversion von Hemiendoprothese auf eine Totalendoprothese)

- im Revisionsfall kann komplikationslos auf eine anatomische oder inverse Schaftprothese gewechselt werden

- Möglichkeit des minimalinvasiven Zugangs

\section{CUP-Prothesen}

Seit 1981 kam der in Lund, Schweden, entwickelte humerale Oberflächenersatz zur Anwendung (Scan Shoulder, Fa. MITAB, Sjöbo). Dabei handelte es sich um eine hemisphärische und $1,7 \mathrm{~mm}$ dicke Kappe, die nach Oberflächenbearbeitung auf den belassenen Humeruskopf zementiert wurde. Der für die rheumatische Schulter entwickelte Gelenkflächenersatz sollte den Eingriff am proximalen Humerus minimieren und die Anatomie möglichst erhalten [11]. Damit wollte man die Nachteile der Stielendoprothesen v.a. bei jungen Patienten mit rheumatoider Arthritis, wie sie nach Prothesenlockerung mit Knochensubstanzverlust im Schaftbereich auftreten kön- 


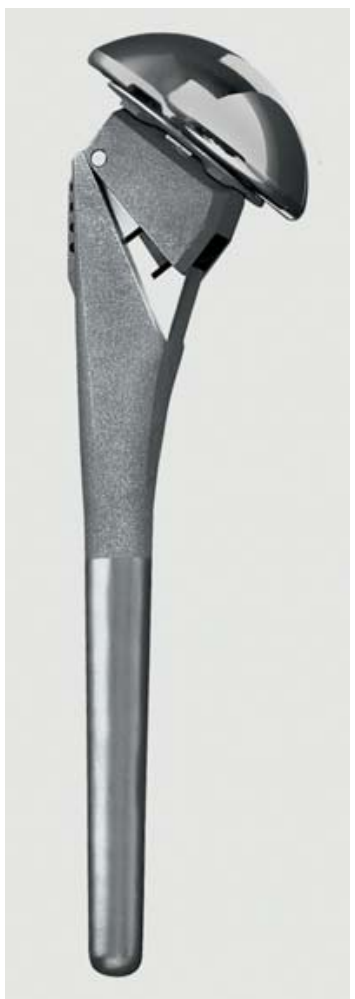

Abb. 3 Humersukopfprothese der vierten Generation. Univers II Prothese (Fa. Arthrex).

nen, umgehen. Steffee und Moore [117] hatten bereits 1984 über ihre Ergebnisse mit einem Cup-System bei der rheumatoiden Arthritis der Schulter berichtet. Zum Durchbruch verholfen hat diesem System Copeland in England (Fa. Biomet; Abb.5), der die Indikationen auch auf Omarthrosen ausgedehnt hat. Mit einem modifizierten Cup-Design mit zentralem, zylindrischem Konus, der im Kopf zementfrei verankert wird, kann Copeland über 17-Jahres-Ergebnisse berichten $[12,13]$.

In Deutschland wurde 1996 ein ähnliches Cup-System mit zentral zylindrischen Zapfen von Rüther (Durom Schulter-Cup, Fa. Zimmer) inauguriert. Der prinzipielle Vorteil bei diesen Cup-Implantaten ist darin zu sehen, dass gerade beim jüngeren Patienten nach langen Standzeiten der Cup-Prothese eine einfache Rückzugsmöglichkeit für die allfällige Wechseloperation gegeben ist. Der Indikationsbereich für dieses Implantat besteht bei der unkomplizierten Omarthrose, Omarthritis (chronische Polyarthritis) und der Humeruskopfnekrose.

Einwände gegen diese Systeme ergeben sich daraus, dass dieses Verfahren konzeptionsbedingt einen gleichzeitigen oder später notwendigen Pfannenersatz

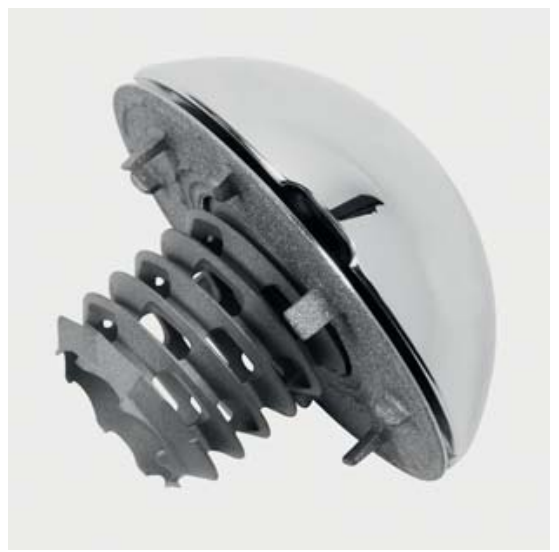

Abb. 4 Schaftfreier (metaphysär verankerter) Humeruskopfersatz. Eclipse Prothese (Fa. Arthrex).

durch Belassen der Kopfkalotte deutlich erschwert und aufgrund der operationstechnischen Schwierigkeit der Pfannenexposition eine ggf. notwendige primäre Pfannenversorgung unterbleibt. Ursprünglich waren die Copeland-CupProthesen (Fa. Biomet) overconstrained konstruiert, um den Knorpelverbrauch von $4 \mathrm{~mm}$ zu kompensieren. Das führte aber häufig zu vermehrter Lateralisation mit eingeschränkter Bewegungsfreiheit. Moderne Cup-Implantate weisen eine geringere Kopfhöhe auf (Fa. Tornier, Fa. Synthes) und bieten anatomische Kalottengrößen an.

\section{Verankerung der Standardschaftprothese}

Die von Neer 1951 inaugurierte Kopfprothese fand ursprünglich ihre Anwendung als zementfreie Frakturprothese [14]. Mit der Einführung des Knochenzements und nach Änderung des Prothesendesigns (Neer-II-System) war die Verwendung als Universalprothese vollzogen (Neer 1974). Die Neer-II-Prothese musste zementiert werden, da sowohl vom Instrumentarium als auch vonseiten der fehlenden Oberflächenbearbeitung eine Press-fit-Fixation nicht vorgesehen war. Versuche, nicht oberflächenbearbeitete Schäfte in Press-fitTechnik zu implantieren [15], haben sich nicht bewährt [16,17]. Eine optimale Zementmanteldicke von 2 bis $3 \mathrm{~mm}$ sollte auch am Humerus angestrebt werden, um die Festigkeit des Zement-KnochenInterfaces zu erhöhen [18].

Moderne Zementiertechniken der 3. Generation unter Verwendung von Markraumstoppern, Jet-Lavage, retrograder Zementapplikation mittels Spritze und Druckzementierung werden nicht generell bejaht. Nach Ansicht von Rockwood

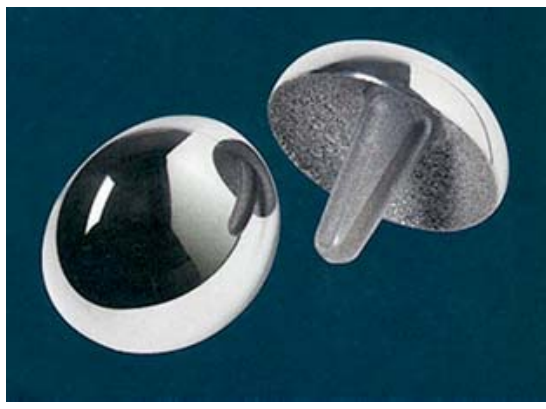

Abb. 5 Cup Prothese. Copeland-Cup (Fa. Biomet Deutschland $\mathrm{GmbH}$ ).

und Matsen verbietet sich am proximalen Humerus der Einsatz eines Zementstoppers und der Druckzementierung. Der Zement wird mittels Finger-Packing nur so weit proximal eingebracht, bis eine Rotation der Stielprothese verhindert wird, da das Erzielen einer proximalen Verankerung bei gleichzeitiger Rotationsstabilität im Vordergrund steht [19].

Rostfreie Chrom-Kobalt-Legierungen (CrCo) müssen auch heute als Standard für zementierte Komponenten gelten. Titan als Prothesenmaterial für zementierte Stiele war im Bereich der Hüftendoprothetik in Verruf gekommen, obgleich auch durchaus gute 10-Jahres-Resultate erzielt wurden [20-22]. Das Problem ist nicht der Werkstoff Titan an sich, sondern die Oberflächenbeschaffenheit des Implantats [23]. Seit Mitte der 90er-Jahre werden die oberflächenpolierten Titanschäfte der Aequalis-Prothese (Fa. Tornier) an der Schulter zementiert implantiert, ohne dass von Lysesäumen oder Lockerungen berichtet wurde. Möglicherweise kommen glatte Titanstiele mit dünneren Zementschichten aufgrund eines niedrigeren E-Moduls mit resultierender geringerer Zementbeanspruchung aus [24].

\section{Press-fit-Design}

Das Durchschnittsalter der Patienten zum Zeitpunkt der Schulterprothesenimplantation liegt etwa um 10 Jahre unter dem der Patienten bei Hüftprothesenimplantation. Mit entsprechend längeren Standzeiten ist zu planen. Neben der dauerhaften Prothesenverankerung muss für die Langzeitstabilität des Gelenkersatzes der Schulter die muskuläre Integrität der Rotatorenmanschette erhalten sein, die jedoch besonders degenerativen Prozessen unterworfen ist. Besteht die Notwendigkeit, eine zementierte Stielprothese wechseln zu müssen, 
so ist es nahezu unmöglich, den Schaft zu entfernen, ohne den proximalen $\mathrm{Hu}-$ merus zu osteotomieren [25]. Dies hat Folgen für die Verankerung des Revisionsschafts.

Somit sind zementierte Verankerungen von Endoprothesen bei jungen aktiven Patienten und bei Revisionen unbefriedigend.

Voraussetzung für eine Primärstabilität ist die mechanische Ruhe am Interface, d.h. Eliminierung von Relativbewegungen. Hierfür sind Oberflächenkonfiguration und Formgebung von entscheidendem Einfluss. Ebenso wie an der Hüfte haben sich vollstrukturierte, grob-poröse Oberflächen nicht bewährt. Speziell bis zur Schaftspitze oberflächenbearbeitete Schäfte, die auch in der Diaphyse ossär eingebaut werden, verhalten sich beim Prothesenwechsel wie eine zementierte Prothese und sind kaum zu entfernen [25]. Die Entwicklung führte zu teilstrukturierten Implantaten mit proximaler Oberflächenstrukturierung, um eine distale Krafteinleitung zu minimieren. Weitere Formen der Oberflächenbearbeitung sind die mikroporöse Raustrahlung (z.B. Ra $=4$ bis $6 \mu \mathrm{m}$ ) und die osteokonduktive Hydroxylapatitbeschichtung mit dem Ziel der knöchernen Integration ohne bindegewebige Zwischenschicht.

Die Formgebung der Stielprothesen war im Schulterbereich bisher keinen vergleichbaren Änderungen unterworfen wie im Hüftbereich. Der Stiel determiniert die Insertionstiefe und die Rotation, die von großer Bedeutung für die Rotationsstabilität des Implantats ist. So verwendet z. B. die Univers-Stielprothese (Fa. Arthrex) einen konischen Geradstiel mit rechteckigem Querschnitt zur primären metaphysären Press-fit-Verklemmung, wobei die seitlich abgeflachte Finne die Rotationsstabilität erhöht. Distal können zylindrische Stielprothesen zu einer stabileren Verklemmung als abgeflachte führen, da der Markraum diaphysär rund bis elliptisch geformt ist [26]. Die Effektivität einer metaphysären Press-fit-Verklemmung ist mittlerweile auch in klinischen Studien bestätigt [27].

Eine optimale distale Stielverklemmung trägt mit nur $30 \%$ zur Rotationssicherheit des Implantats bei [28].

Der exakt gefräste Markraum erlaubt nur noch die Verstellung der Prothesenhöhe [9]. Somit kommt der Markraumbearbei- tung eine zentrale Bedeutung zu, da nur durch eine ideale Abstimmung der Fräsgeometrie zur Implantatgeometrie der Press-fit erzielt wird. Die akkurate Markraumpräparation bedingt eine verbesserte primäre Fixation. Ein zu hohes Einsetzen des Stieles in Relation zu den Tubercula muss vermieden werden, da hierdurch eine Überspannung der inferioren Kapsel erfolgt, mit Limitierung der Abduktion und Kranialisierung des Drehzentrums und damit Verringerung des Kraftarms der Rotatorenmanschettenmuskeln mit sekundärem Versagen [29].

An der Schulter sind nach eigener Erfahrung bisher bei Press-fit-fixierten Stielprothesen nur sehr selten Oberarmbeschwerden durch sog. Stress Shielding mit proximalem Knochensubstanzverlust aufgetreten.

Aufgrund sehr niedriger und klinisch nicht relevanter Schaftlockerungsraten sind die Standardstiele bisher nicht von anatomischen oder individuell angefertigten noch modularen Schäften abgelöst worden. Im Gegenteil, die Entwicklung geht zu „Short-stem“-Implantaten bis hin zu den schaftfreien Implantaten der sog. 5. Generation.

\section{Pfannenersatz}

Die Schultergelenkpfanne ist konzentrischen und exzentrischen Belastungen im Sinne von Kompressions- und Scherkräften ausgesetzt, die aufgrund der kleinen Belastungsfläche und dem langen Hebelarm unter statischen unbelasteten Bedingungen bereits $89 \%$ des Körpergewichts betragen [30] und unter Belastung bei gleichzeitiger Bewegung des Armes weit über das Körpergewicht hinausgehen [31]. Die Ungleichheit der Gelenkflächengröße führt zu einem Belastungsübergewicht an der Pfanne, da sie einem längeren Belastungszyklus (duty cycle) als der größere Humeruskopf ausgesetzt ist.

\section{Allgemeine Konstruktionsmerkmale des Pfannenersatzes}

Die Konformität der Gelenkpfanne mit dem Kopfradius, d.h. die Übereinstimmung von Kopf- und Pfannenradius verbessert den Gelenkkontakt, verschlechtert aber im Gegenzug die glenohumerale Beweglichkeit [32] und führt zum Polyethylenabrieb [33]. Unter dynamischen Verhältnissen führt jede Translation des Humeruskopfs zu einer exzentrischen Belastung der Gelenkpfanne, mit hohem
Kontaktdruck am Pfannenrand, asymmetrischem Polyethylenverbrauch und Polyethylenkaltfluss. Es entsteht das sog. Rocking-Horse-Phänomen, das Matsen zuerst beschrieb [19]. An der gegenüberliegenden Seite des maximal exzentrisch belasteten Pfannenrands beginnt die Lockerung, was am radiologisch sichtbaren Lockerungssaum erkennbar wird. Exzentrische Dysbalancen entstehen nicht nur durch vertikal-kranial gerichtete Scherkräfte beim Rotatorenmanschettendefekt, wie z.B. im Rahmen der rheumatoiden Arthritis, sondern auch bei exzentrisch horizontaler Belastung, wie sie bei Instabilitäten oder bei der Omarthrose mit hinterer Subluxation des Kopfes auftreten können.

In einer Finite-Elemente-Studie konnten Swieszkowski et al. [34] nachweisen, dass am Glenoid Spitzenbelastungen bis $25 \mathrm{Mpa}$ auftreten. Dieser Wert liegt über der Belastbarkeit des Polyethylen als Werkstoff, sodass mit dem Auftreten von Abrieb und sog. Kaltfluss (cold flow), also einer schleichenden Verformung, gerechnet werden muss. Mehr konforme Implantate zeigen geringere Kontaktdrücke, was theoretisch zu niedrigeren Abriebraten und einer geringeren Gefahr des Auftretens von Kaltfluss führt. Theoretisch zeigen Metalback-Implantate höhere Kontaktdrücke am Polyethylen bei gleicher Polyethylendicke, was sich theoretisch in schlechteren Abriebwerten niederschlagen würde.

Der Vorteil einer flacheren Gelenkpfanne mit bis zu 6 mm größerem Kurvenradius ist in einer verbesserten Translation ohne Overbacking des Pfannenrands und vermindertem Polyethylenverbrauch zu sehen [35,36]. Mit zunehmendem Mismatch (vergrößerter Radius der Pfannenoberfläche gegenüber dem Humerus) erhöht sich der Anpressdruck auf bis zu 300\%. Dieser Effekt wird noch verstärkt durch pathologische Retroversionswerte. Des Weiteren nimmt nicht nur die Belastung im Polyethylen sondern auch im Zementmantel zu. Über einem Mismatch von $6 \mathrm{~mm}$ wird die Festigkeit des Materials durch Kräfte, die bei der einfachen unbelasteten Abduktion entstehen, überschritten [37,38]. Durch die genannten Faktoren ist die Lockerungsgefahr für weniger konforme Pfannen geringer als für konforme Pfannen [39-41].

Die heutigen auf dem Markt befindlichen Glenoidkomponenten weisen daher einen höheren Mismatch auf. 
In einer Untersuchung an ausgebauten Pfannen konnte Nho [42] nachweisen, dass es bei Pfannen mit gleichen Radien zu höherer Versagerquote gekommen ist als bei solchen mit größerem Mismatch.

Bigliani und Flatow vereinen mit ihrer Pfanne (Fa. Zimmer) einen Kompromiss zwischen Konformität und Non-Konformität: Das Pfannenzentrum ist konform, die Randzone jedoch nicht konform ausgearbeitet.

Zusammengefasst fehlen immer noch wissenschaftliche Daten, um eine abschließende Einschätzung der idealen Implantatgeometrie zu untermauern. Insbesondere das etablierte Prinzip der Non-Konformität wird durch die Analyse von Glenoidkomponenten nach Wechseloperationen, die regelhaft ein „Einlaufen“" zu konformen Radien zeigen, infrage gestellt [43].

Die Ausformung der Pfannenrückseite ist Gegenstand unterschiedlicher Betrachtung. Die meisten Hersteller verwenden der natürlichen Pfannenform angepasste konvexe Formen. Konvexe Formgestaltung ist knochensparend, lässt sich mit entsprechenden Fräsen im Pfannenlager einfach einfräsen und besitzt im Vergleich zur planen Rückseite eine größere Auflagefläche. Einer exzentrischen Scherbelastung wirkt eine konkave Pfannenauflage durch relativ größere Auflagefläche und damit größerem Widerstand entgegen. Zusätzlich werden die exzentrisch auftretenden Randbelastungen gleichmäßiger auf das knöcherne Pfannenlager übertragen und reduzieren die Gefahr einer vorzeitigen Lockerung [44].

Plane Rückflächen (Aequalis, Fa. Tornier; Select Shoulder, Fa. Sulzer) wurden unter der Überlegung konzipiert, dass beim dorsalen Gelenkflächenverbrauch für die Pfannenpräparation nur ventral der knöcherne Pfannenrand abgetragen werden muss. Im mechanischen Vergleichstest sind konvexe Rückflächen stabiler als flache $[39,45]$.

Noch größere Verankerungsstabilität verspricht man sich von stufenförmigen Rückflächen, die durch Vergrößerung der Auflagefläche und durch tiefere Verankerung besser gegen den RockingHorse-Mechanismus schützen (Univers, Fa. Arthrex; Abb. 6).

Wichtiger als die Formgebung der Pfannenrückfläche ist die passgenaue, d.h. perfekte Bearbeitung der Glenoidpfanne mit größtmöglicher Kongruenz von Pfannenimplantat und Pfannenlager.

Je passgenauer die Pfannenpräparation, desto höher die Formstabilität und entsprechend geringer das Lockerungsrisiko [44].

Aufgrund der räumlichen Enge ist es insbesondere bei ungleichmäßigem Pfannenverbrauch mit dorsaler Pfannenabflachung technisch schwierig, eine absolut kongruente Auflagefläche zu präparieren. Das setzt ein geeignetes Instrumentarium für die Pfannenbearbeitung voraus. Spezielle Pfannenfräsen müssen im Pfannenzentrum gesichert sein oder einen selbstzentrierenden Schliff aufweisen.

Eine zu aggressive Pfannenbearbeitung kann zu einem ungleichmäßigen Verlust der subchondralen Kortikalis mit Freilegen der Spongiosa führen. Dies schafft Belastungsflächen mit verschiedener Belastbarkeit und in der Folge Lockerung der Pfanne.

Die Orientierung der Glenoidkomponente - Realignement - in Bezug auf die Ante-/Retroversion in der Horizontalebene ist besonders wichtig, um Stress auf die implantierte Pfanne zu reduzieren und damit der Lockerung vorzubeugen. Künstliche Veränderungen der Pfannenversion führen zu einer pathologischen Kopftranslation von $0,5 \mathrm{~mm}$ pro $1^{\circ}$ Winkeländerung.

Eine „kompensatorische“ Korrektur der Humeruskopftorsion kann eine abnormale Pfannenversion nicht korrigieren [46-48].

Dies hat erhebliche Konsequenzen für die Implantationstechnik, dass die physiologische Retroversion der Glenoidfläche wiederhergestellt wird. Dies trifft nach Erachten der Autoren mit gleicher Konsequenz auch auf die Inklinationsneigung der Pfanne in der Sagittalebene zu.

Sowohl die pathologische Retroversion als auch eine pathologische Inklination der Pfanne müssen korrigiert werden!

Wird eine zu großflächige Glenoidkomponente eingebaut, so führt dies zu einem glenohumeralen Impingement. Dabei kommt es in der $0^{\circ}$-Stellung zu einem Kontakt zwischen Calcar humeri und unterem Pfannenrand bzw. in Ab-

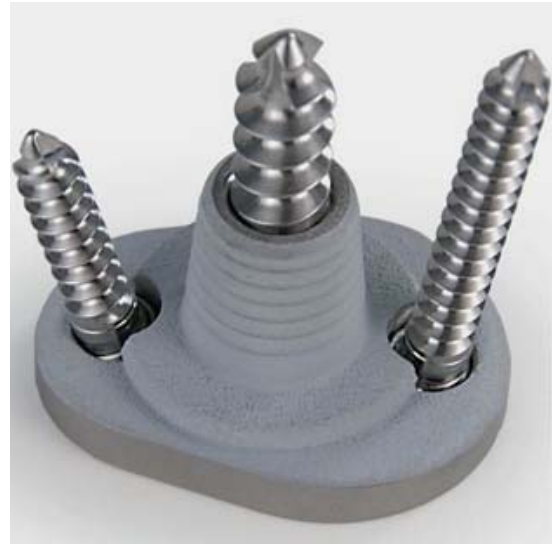

Abb. 6 Stufenförmige Rückfläche einer zemenfreien Metal-back Glenoidkomponente (Universal Glenoid, Fa. Arthrex).

duktion zwischen Rotatorenmanschette und Oberrand der Pfanne. Das Resultat ist die frühe Lockerung der künstlichen Pfanne [49] im Sinne eines kraniokaudalen Rocking-Horse-Phänomens. Weiterhin wird die biomechanische Gesamtsituation (Kräfteverteilung und Kontaktpunkt) durch eine zu groß gewählte Komponente ungünstig beeinflusst [50].

\section{Zementierte Glenoidkomponenten}

\section{Kielglenoidkomponente}

1973 entwickelte Neer (Neer 1974) eine Polyethylenpfanne, nachdem Zippel (1972) und Kenmore (1973) eigene Polyethylenglenoidkomponenten mit der Original-Neer-Kopfprothese kombiniert und als Totalprothesen implantiert hatten. Gleichzeitig veränderte Neer die Schaft- und insbesondere die Kopfgeometrie (Neer-II-System), um sie auf die Polyethylenkomponente abzustimmen. Die Pfanne wies den gleichen Krümmungsradius von $44 \mathrm{~mm}$ wie die Kopfkalotte auf und wurde mit Methylmetacrylat einzementiert. Bis zur Markteinführung 1982 wurde das Design insgesamt 5-mal geändert [15]. Die Konformität der Krümmungsradien war eine der Ursachen für das häufige Auftreten von Lockerungssäumen an den Pfannen. Heutige Pfannenkomponenten weisen meistens nicht konforme Krümmungsradien auf, um einen verbesserten RollGleit-Mechanismus zu gewährleisten.

Das von Neer eingeführte trianguläre Kieldesign zur Pfannenverankerung bleibt das weitverbreitetste und gilt als Goldstandard. Es besteht eine Vielzahl von Kielvarianten mit gering unterschiedlichem Stressverteilungsmuster 


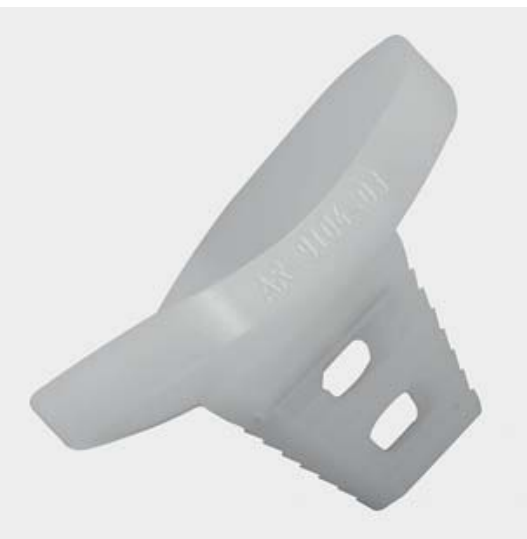

Abb. 7 Zementierte Kiel-Glenoidkomponente mit Aussparung im Kielbereich (Fa. Arthrex).

[51]. Neuere Varianten zeigen unterschiedliche Hinterschneidungen oder Ausspaarungen im Kielbereich um eine verbesserte Zementverankerung zu gewährleisten (Abb.7). Resch konzipierte ein Modell, bei dem die Kielposition in der Koronarebene exzentrisch nach vorne, vor die Mittellinie versetzt wird, um eine bessere Knochenverankerung zu erlangen. Experimentell konnte gezeigt werden, dass die Beanspruchung am Zement-Implantat-Interface bei maximaler Belastung in Abduktion und Flexion geringer ist gegenüber einem Implantat mit zentral sitzendem Kiel [52].

Wichtig ist eine Präparation und Zementiertechnik, die einen gleichmäßigen Zementmantel und so wenig wie möglich sog. Radiolucent Lines produziert. Die von Neer empfohlene Kürettage zur Ausräumung der Spongiosa, um Platz für den Knochenzement zu schaffen, ist zugunsten einer Spongiosakompaktion verlassen worden. Das Einpressen von Knochen in den Kielschlitz (bone compaction) verbessert dabei offenbar die Zementierung [45]. Die Zementierung mit Finger-Packing-Technik zeigt im Vergleich zur Einbringung mittels Zementspritze in $47 \%$ Zementlücken [53].

\section{Peg-Glenoidkomponente}

Alternativ werden Pfannensysteme mit Verankerungszapfen (Pegs) statt eines Kieles bereitgehalten (Global Shoulder, Fa. DePuy; Anatomica, Fa. Zimmer). Theoretisch ist dabei von Vorteil, dass weniger Knochen entfernt werden muss und mehr Oberflächenkontakt für den Zement bei jedoch geringerer Zementmasse zur Verfügung steht [54]. Mehrere kleinere Pegs bieten eine größere Stabilität gegen Scherbelastung und gleich-

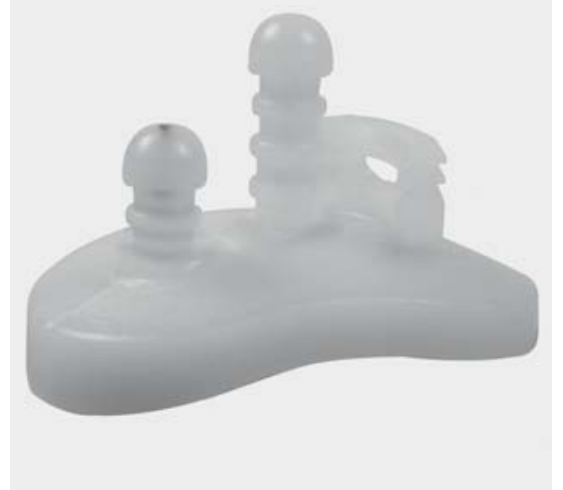

Abb. 8 Zementierte Kiel-Peg-Glenoidkomponente nach Romeo (Fa. Arthrex).

mäßigere Stressverteilung als wenige und größere Zapfen [55].

Potenziell besteht die Gefahr, bei der Bohrung der Zapfenlöcher das Glenoid zu fragmentieren. Auch bei der Pfannenbearbeitung für die Kielaufnahme kommt es zur potenziellen Schwächung der Pfanne, insbesondere durch Knochenverlust. Bleibt bei der Pfannenpräparation der subchondrale Knochen unbeschädigt, besteht experimentell jedoch nach einer Finiten-Elemente-Analyse kein Unterschied zwischen Kielund Zapfendesign [51]. Im mechanischen Labortest konnte Gerber dies widerlegen und findet für ein konturiertes Peg-Design im Vergleich zu den Kielpfannen eine signifikant höhere Belastungsstabilität. Wenn die Polyethylenrückfläche rau bearbeitet ist, dann erhöht dies zudem die Verankerungsstabilität [39].

Röntgendurchlässige Säume (radiolucent lines) und inkomplette Verankerung der Komponenten sind beim Kieldesign hochsignifikant häufiger als bei der Peg-Pfanne [56-58]. Eine neue Variante einer Peg-/Kielpfannenkombination soll die Vorteile beider Komponenten miteinander vereinen (Abb. 8; Univers $^{\mathrm{TM}}$ II, Fa. Arthrex).

Als Modifikation der zementierten PegPfanne entwickelte Hertel eine sog. Hybridpfanne (Epoca, Fa. Synthes), bei der nur die Verankerungszapfen in Hohlschrauben einzementiert werden, die wiederum zementfrei in der Spongiosa verankert sind.

Insgesamt kommt es bei Verwendung der zementierten Polyethylenpfannen zu einem trabekulären Stressvertei- lungsmuster, das dem eines intakten Glenoids entspricht [59].

Unabhängig von den Designcharakteristika ist allen zementierten Varianten der Nachteil des Zementierens gemein. Hitzeentwicklung und fehlerhafte Zementiertechnik bei unterbleibender Bluttrockenheit und unvollständiges Zementieren des Kielschlitzes und der Polyethylenauflagefläche führen zur Zementlockerung bzw. zum Zementbruch.

Eine verbesserte Zementiertechnik mit Auftragen von Zement auf die Rückfläche des Implantats und Einpressen von Zement in die Peg-Löcher kann das Auftreten von Röntgensäumen signifikant verringern [53,60-62].

\section{Unzementierte Glenoidkomponenten}

Metalback-Glenoidkomponenten mit Schraubverankerung

1976 wurde von English und McElwain (McElwain und English 1987) erstmals eine unzementierte Schulterpfanne entwickelt, die aus einem Polyethyleneinsatz und einem oberflächenbearbeiteten Metallträger mit Schraubenfixierung bestand. Cofield (1994) verfügt seit 1981 über die längste Erfahrung mit zementfreien Schraubpfannen. Sein Konzept besteht aus einem Metallträger mit einem zentralen Zapfen, der in das Pfannenlager gefräst und mit 2 zusätzlichen Schrauben im Glenoid stabilisiert wird. Auf dem Metalback wird eine Polyethylenkomponente aufgebracht. Monoblock-Metalback-Prothesen werden auch von weiteren Herstellern angeboten (Fa. Biomet, Fa. Kirschner, Fa. Lima). Eine sichere primäre Verankerung mit möglichst geringen Mikrobewegungen am Knochen-Implantat-Interface begünstigt die knöcherne Einheilung der Rückfläche des Metalbacks [63].

Experimentell liegt das Rocking-HorsePhänomen der zementlosen Pfannen nur gering über dem der Polyethylenpfannen [39].

Ein anderer Einwand betrifft die Bauhöhe von bis zu $10 \mathrm{~mm}$ für den zementfreien Pfannenersatz, was nicht nur zu einer Lateralisierung mit zu hoher Vorspannung für den Weichteilverschluss und damit zur Bewegungseinschränkung führt, sondern auch mit einer höheren Exzenterbelastung verbunden ist. Die neueren Systeme warten mit Stärken von 6 mm (Univers, Fa. Arthrex) auf, wo- 
bei die Dicke des Inlays davon $4 \mathrm{~mm}$ beträgt. Ein weiterer Nachteil besteht in der Möglichkeit der Dissoziation des Polyethyleneinsatzes. Sonnabend berichtet bei 50 Fällen von 4\% [66], im eigenen Krankengut weist die Dissoziationsrate $1 \%$ bei 284 Fällen auf [65].

Metalback-Glenoidkomponente mit Hohlschrauben- und Hohlzylinderprinzip

Mit dem Ziel, ohne Auffräsen oder Setzen von Bohrkanälen eine intakte Subchondralschicht zu belassen, wurde zur Verankerung des Metalback eine selbstschneidende, $13 \mathrm{~mm}$ und für Revisionsfälle eine $19 \mathrm{~mm}$ starke Hohlschraube (engl. cage screw) angewandt, die in den spongiösen Knochenkanal des Glenoids eingedreht wird und mit ihrem Schraubenkopf das Metalback fixiert. Randelli verwendet statt einer Hohlschraube einen Hohlzylinder (Fa. Lima). Der zentrale Knochenzylinder im Kern der Hohlschraube oder des Metallzylinders bleibt dabei erhalten und kann als Abstützung dienen. Diese Systeme bieten nicht nur ein Maximum an Verankerungsfläche für die Osteointegration, sondern sichern auch gegen exzentrische Belastungen entgegen der Zugrichtung.

Unzementierte Pfannensysteme mit Metal-backing haben den theoretischen Vorzug, eine Osteointegration zu ermöglichen und damit längere Standzeiten zu erzielen. Eine Finite-Elemente-Analyse aus der Mayo-Klinik konnte zeigen, dass die Gesamtstressbelastung im subchondralen Knochen mit unzementierten Metal-backed-Komponenten viel geringer als mit zementierten Polyethylenpfannen ist [59], und dies besonders bei exzentrischer Belastung. Damit bestätigt sich die Finite-Elemente-Untersuchung von Friedman [51], der mit zementfreien Systemen eine physiologischere, d.h. gleichmäßigere Stressverteilung nichtaxialer Kräfte gefunden hatte. Zudem führen unzementierte im Vergleich zu zementierten Komponenten $\mathrm{zu}$ einer stabileren Fixation im Knochen (Stone et al. [59]). Dies gilt allerdings nicht für alle Prothesendesigns in gleichem Maß [66].

Der kritische Bereich für zementfreie Pfannen ist der Übergang zwischen Polyethylen-Inlay und Metallpfanne. Hier kommt es zu hohen Stressbelastungen; dies ist der Ausgangspunkt für das Versagen des Polyethylens. Wie hoch die Belastung sein muss, um eine definitive Po-

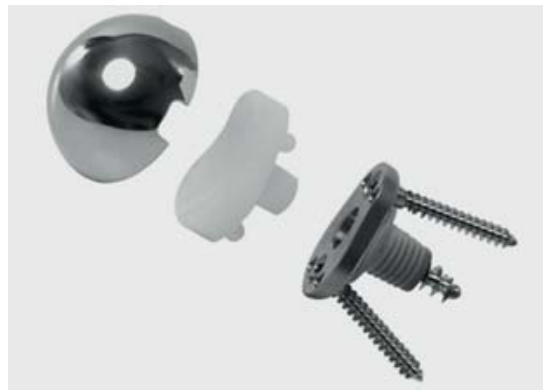

Abb. 9 Zementfreie konvertierbare Universal Glenoidkomponente (Fa. Arthrex).

lyethylenschädigung auszulösen, lässt sich bisher mit zweidimensionalen mathematischen Modellen noch nicht berechnen [59]. Die geringe Rigidität des Metalback scheint die Übertragung der Kräfte vom Polyethylen zur Metallpfanne $z u$ begünstigen.

\section{Zementfreie Glenoidkomponente mit Press-fit-Verankerung}

Copeland (Copeland ${ }^{\mathrm{TM}}$, Fa. Biomet) verzichtet auf eine Schraubenfixation des Metallträgers und versieht den Metallträger mit einem zentralen Konus, der im Glenoid [12,67] press-fit verankert wird.

\section{Universalglenoidkomponente}

Mit zunehmender Verbreitung der Schulterprothetik und bei Überalterung der Bevölkerung besteht zunehmend die Notwendigkeit, Pfannensysteme anzubieten, die sowohl der anatomischen als auch der inversen Verwendung Rechnung tragen. Konzeptionell handelt es sich dabei um zementfreie MetalbackSysteme mit zentralem Konus und winkelstabiler Verschraubung, auf die entweder Polyethylen-Inlays oder Glenosphären aufgesetzt werden können (Abb. 9; Fa. Arthrex, Fa. Zimmer).

Für alle Pfannenersatzsysteme ist letztlich entscheidend, wie stabil ihre Verankerung ist. Das ist auch eine Frage des Pfannendefekts und der Knochenqualität, wie genau die Komponenten ausgerichtet sind und wie weit sie eine anatomische Gelenklinie rekonstruieren. Dies gelingt prinzipiell mit zementierten und zementfreien Glenoidkomponenten. Das Hauptproblem stellt die physiologische Rezentrierung der Kopfkalotte dar. Bei persistierender Subluxationsstellung der Kopfprothese ist eine Lockerung aller Pfannensysteme wahrscheinlich.

\section{Indikationen zur anatomischen Schulterprothetik}

Die Indikation zur Implantation einer Schulterprothese ist gegeben bei einer Arthrose unterschiedlicher Ätiologie mit einer Gelenkspaltbreite von weniger als $3 \mathrm{~mm}$, einer hinteren Pfannenabflachung in Kombination mit klinisch mittels Schmerzmedikation nicht mehr beherrschbaren Belastungs- und Ruheschmerzen mit einer zunehmender Bewegungseinschränkung mit einer kritischen Abduktion von weniger als $90^{\circ}$, einer Außenrotation von weniger als $20^{\circ}$ und einer Innenrotation von weniger als bis LWK 5. Im Folgenden werden die Indikationen entsprechend der Ätiologie der Arthrose aufgeführt.

\section{Primäre Omarthrose}

Aus 3 pathomechanischen Gründen besteht die Indikation zur Implantation einer Totalprothese [68]:

- Verlust der posterioren Glenoidkonkavität

- Verlust der Zenterposition für den Kopf durch Abflachung der Pfanne

- persistierende humerale posteriore Subluxationsstellung

Orientiert man sich an der Einteilung der Pfannenarthrose nach Walch, so sehen wir die in Tab. 1 zusammengefasste Differenzialindikation.

Bei dysplastischer Pfanne (Typ C) besteht eine Kontraindikation zum Einbau der Totalprothese, weil die hochpathologische Retro- und Inklinationsfehlstellung keine dauerhafte Pfannenimplantation zulässt.

Wird nur eine Hemiarthroplastik durchgeführt, muss mit $51 \%$ subjektiv unbefriedigenden Ergebnissen und einer Revisionsrate von $18 \%$ gerechnet werden [69]. Vergleicht man die Ergebnisse der Hemiarthroplastik bei konzentrisch intakter mit dorsal verbrauchter Pfanne, so kommt man nur bei konzentrisch erhaltener Pfanne (entspricht Pfannentyp A1 nach Walch) auf zuverlässig gute Ergebnisse [70]. Die schlechtesten Ergebnisse bei der Omarthrose erzielt man bei Implantation einer Hemiendoprothese in Kombination mit hinterer Pfannenerosion [71]. Zudem besteht nach 5 Jahren Standzeit einer Hemiendoprothese das Risiko einer Verschlechterung des Ergebnisses aufgrund eines progressiven Pfannenverbrauchs [72]. 
Tab. 1 Differenzialindikationen zur Schulterendoprothetik bei Omarthrose.

\begin{tabular}{ll}
$\begin{array}{l}\text { Pfannentyp nach } \\
\text { Walch [87] }\end{array}$ & Implantationsart \\
\hline A1 & Hemiendoprothese \\
\hline A2 & Totalendoprothese \\
\hline B1 und B2 & Totalendoprothese \\
\hline C & Hemiendoprothese
\end{tabular}

Tab. 2 Differenzialindikationen zur Schulterprothetik bei avaskulärer Humeruskopfnekrose.

\begin{tabular}{ll}
$\begin{array}{l}\text { Nekrosestadium } \\
\text { nach Cruess [118] }\end{array}$ & Implantationsart \\
\hline II & Hemiendoprothese \\
\hline IV & Hemiendoprothese \\
\hline V & Totalendoprothese
\end{tabular}

\section{Avaskuläre Humeruskopfnekrose}

Die Indikation zum hemiendoprothetischen Gelenkersatz ist in den Stadien III und IV gegeben, da per definitionem noch keine glenoidale Beteiligung vorliegt. Im Stadium V ist ein zusätzlicher Pfannenersatz erforderlich, um die Kongruenz der Gelenkflächen wiederherzustellen (Tab. 2).

\section{Posttraumatische Arthrose}

Die Indikation sowie die Implantatwahl richten sich bei der posttraumatischen Arthrose (chronische Fraktur) nach der Klassifikation nach Boileau.

Um diese Frakturfolgen auch prognostisch einstufen zu können, hat Boileau eine nach dem Schweregrad abgestufte Klassifikation der chronischen Humeruskopffraktur eingeführt [73] (Abb. 10): - intrakapsuläre Formen:

- Typ 1: impaktierte Valgus-/Varusfraktur mit Kalottenkollaps oder Kopfnekrose; eine Tuberkulumsteotomie ist nicht notwendig

- Typ 2: chronisch-verhakte Humeruskopfluxations- oder Luxationsfraktur

- extraartikuläre Formen:

- Typ 3: subkapitale Humeruspseudarthrose

- Typ 4: dislozierte Humeruskopffraktur mit schwerer Fehlstellung oder Pseudarthrose des Tuberculum majus; eine Tuberkulumosteotomie ist notwendig

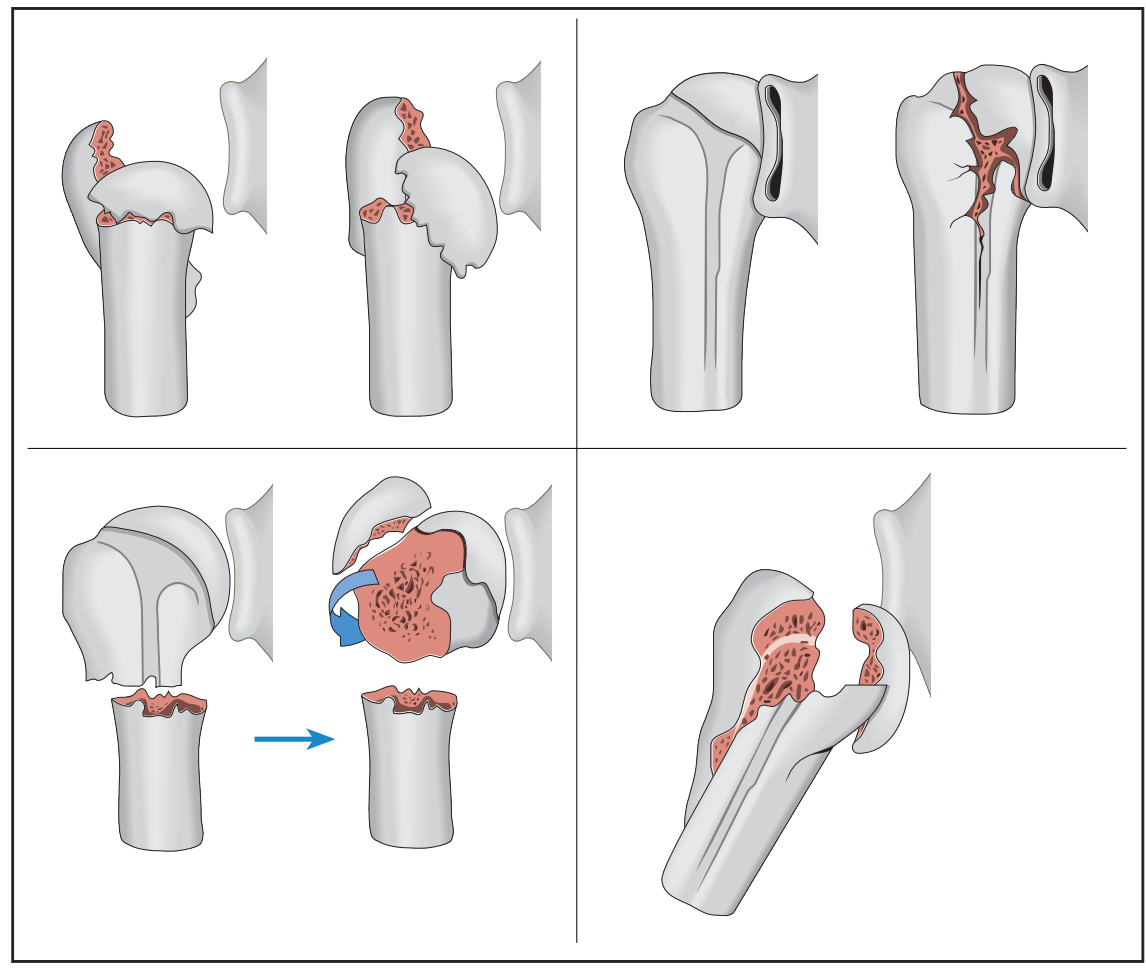

Abb. 10 Klassifikation der chronischen Humeruskopffraktur nach Boileau.

Je höhergradig die Frakturform, desto schlechter die Prognose.

Die chronische Fraktur Typ 1 nach Boileau ist bei intakter oder rekonstruierbarer Rotatorenmanschette die Domäne der anatomischen Schulterprothetik.

Die posttraumatischen Kopfveränderungen führen in einem hohen Prozentsatz zu Kollateralschäden an der Glenoidfläche. Es gelten die gleichen Grundsätze zur Implantation einer Gelenkpfanne wie bei den Omarthrosen. Sechs Monate nach Trauma ist der Knorpelschaden so ausgeprägt, dass eine Pfannenkomponente eingebaut werden muss [74]. Ausnahmen von der Regel sind extrem kontrakte Gelenke mit sehr engen Gelenkverhältnissen. Wenn eine Pfanne nur unter Inkaufnahme eines aufgehobenen Gelenkspiels und einer unter Spannung stehenden Rotatorenmanschette implantiert wird, führt dies nur zu einer schmerzhaften Schultersteife. Nicht rekonstruierbare Rotatorenmanschettenschäden stellen eine weitere Kontraindikation zur Pfannenimplantation dar. In diesen letzten beiden Fällen wäre die Alternative ein inverses Implantat.

Die Indikation zum Gelenkflächenersatz bei chronisch verhakter Humeruskopf- luxationsfraktur Typ 2 nach Boileau ist gegeben bei:

- > $40 \%$ humeralem Gelenkflächendefekt,

- >6 Monate Dislokationsstellung mit Osteopenie und Knorpelerweichung,

- schmerzhaftem Funktionsverlust,

- gegebener Compliance des Patienten und unauffälligem neurologischen Befund.

Bei sehr lange bestehender chronischer verhakter Luxationsfraktur und fettiger Degeneration der Rotatorenmanschette (> Grad 2 nach Goutallier) ist die Indikation zur Implantation einer inversen Schulterprothese gegeben.

Die subkapitalen Pseudarthrosen (chronische Fraktur Typ 3 nach Boileau) sind bei intakter Rotatorenmanschette eine Domäne der kopferhaltenden Rekonstruktion.

Bei der chronischen dislozierten Humeruskopffraktur mit schwerer Fehlstellung oder Pseudarthrose des Tuberculum majus Typ 4 nach Boileau ist überwiegend bei älteren Patienten die Indikation zur Implantation einer inversen Prothese gegeben.

Kontraindiziert ist die sekundäre Implantation der Frakturprothese bei einer Parese des N. axillaris sowie bei einer 
Armplexuslähmung. Dies ist eine Domäne für die Schulterarthrodese. Des Weiteren gilt auch die floride Infektion als Kontraindikation.

Beim nicht kooperativen Patient ist eine relative Kontraindikation gegeben.

Implantatwahl bei der posttraumatischen Arthrose

Voraussetzung für ein gutes funktionelles Ergebnis ist die anatomische Rekonstruktion des Glenohumeralgelenks mit seinem physiologischen Rotationszentrum durch Wiederherstellung der Kalottenhöhe, des posteromedialen Offsets und des Inklinations- und Retrotorsionswinkels. Dies kann erschwert werden durch Fehlstellung der Tubercula und des Schaftes.

Die 4. Generation der Schaftprothesen erlaubt eine freie Einstellung von Offset, Inklination und Retrotorsion. Die als 5. Generation bezeichneten schaftfreien Humeruskopfprothesen (Eclipse, Fa. Arthrex; T.E.S.S., Fa. Biomet) gewähren darüber hinaus zusätzlich eine voneinander unabhängige Kopf-Schaft-Geometrie, die eine Humerusschaftfehlstellung verzeiht. Schaftfreie Kopfprothesen vermeiden einen valgischen oder varischen Einbau der Stielprothese, wie sie zu 69\% bei chronischer Fraktur beschrieben wurden (Moineau und Boileau [119]). Darüber hinaus erlauben sie einen problemlosen Wechsel auf anatomische oder inverse Schaftprothesen.

Die inverse Prothese kompensiert chronische Rotatorenmanschettenschäden und schwere, nicht rekonstruierbare Fehlstellungen der Tubercula. Tuberculaosteotomien führen grundsätzlich $\mathrm{zu}$ schlechteren Resultaten mit hoher Revisionsrate [74-76].

\section{Rheumatoide Arthritis}

Entscheidend für das operative Vorgehen sind bei der chronischen Polyarthritis die ossäre Destruktion (humeral und v.a. glenoidal) und die frühzeitige Schädigung der Rotatorenmanschette.

Das Ziel sollte sein, den endoprothetischen Gelenkersatz nicht so weit hinauszuzögern, bis die Gelenkpfanne und die Rotatorenmanschette irreparabel zerstört sind.

Die Beurteilung des Zustands dieser beiden Gelenkanteile muss präoperativ durch eine adäquate Diagnostik (NativRöntgen, MRT und CT) vorgenommen werden. Unauffällige Röntgenaufnahmen schließen eine rheumatoide Mitbeteiligung nicht aus. Eine beginnende Gelenkspaltverschmälerung ist oft der Umkehrpunkt zur rapiden Gelenkzerstörung [77].

In den Stadien I und II nach LDE bzw. Stadium 1 nach Lévigne ist ein Gelenkersatz nicht zu diskutieren. Diese Stadien sind die Domäne der arthroskopischen Frühsynovektomie mit anschließender Radiosynoviorthese. Allerdings besteht bei aufgehobenem Gelenkspalt (LDE III, Lévigne 2), Gelenkflächendefekt und intakter Rotatorenmanschette die Indikation zur Totalendoprothese. Ist die suffiziente Funktion der Rotatorenmanschette nicht gesichert, so ist der isolierte Humeruskopfersatz indiziert. Das Endstadium der chronischen Polyarthritis (LDE V, Lévigne 3 ) bietet bei weitgehend zerstörter Gelenkpfanne und ausgedehnter Ruptur der Rotatorenmanschette keine Möglichkeit eines Pfannenersatzes. Ziel ist es, durch einen hemiendoprothetischen Kopfersatz die ansonsten therapieresistenten Schmerzen zu lindern [78]. Alternativ bei noch erhaltenem Glenoid besteht die Indikation zur inversen Totalprothese.

Insgesamt ist das Ergebnis funktionell nicht mit der Omarthrose zu vergleichen, vielmehr muss der Patient darüber aufgeklärt werden, dass im Spätstadium die Schmerzreduktion im Vordergrund der operativen Therapie steht.

\section{Instabilitätsarthrose}

Die Indikation zur Hemiarthroplastik besteht bei:

- Stadium Grad II und III nach Samilson,

- erhaltener Pfannenkonkavität Typ AI nach Walch,

- fehlender humeraler Subluxation,

- erhaltenem Gelenkspalt $>3 \mathrm{~mm}$ in 3 Röntgenprojektionen.

Die Indikation zur Totalprothese ergibt sich bei:

- Stadium Grad II und III nach Samilson,

- Pfannendeformität Typ B Grad I und II nach Walch,

- Subluxationsstellung des Humeruskopfs,

- fehlendem Gelenkspalt in einer von 3 Röntgenprojektionen.

Bei der Implantation einer Schulterprothese aufgrund eine Instabilitätsarthrose müssen jedoch noch weitere Aspekte berücksichtigt werden.

Ist die Arthrose Folge einer chronischen Dislokation, besteht prinzipiell als primäre Pathologie ein Pfannenrandschaden, der die glenoidale Konkavität außer Kraft setzt. Somit ist kausal eine prothetische Pfannenrekonstruktion zur Wiederherstellung der Stabilität zwingend [68]. Geht die Instabilitätsarthrose auf die Folge einer Stabilisierungsoperation zurück, so ist sie auf eine zu straffe Kapselrekonstruktion zurückzuführen. Dies verursacht in der entgegengesetzten Richtung eine Subluxation und sekundären Pfannenschaden durch die exzentrische Pfannenrandbelastung. Aus diesem Grund muss neben dem Kapsel-Release mit Verlängerung der Subskapularissehne und Korrektur der Subluxationsstellung die geschädigte Pfanne in der Regel ersetzt werden (Abb. 11).

Besonders bei der primären Instabilitätsarthrose kann es nach ventralem KapselRelease und Subskapularisverlängerung zu der paradoxen Situation kommen, dass die zugrunde liegende Kapselhyperlaxität wieder zutage tritt und nach Implantation der Prothese eine meist hintere und untere Kapselhyperlaxität mit vermehrtem Gelenkspiel eintritt. Die Prothese lässt sich nach hinten luxieren und zentriert sich nicht selbst. Um dies zu umgehen, beschränkt man das Kapsel-Release nur auf die verkürzte vordere Kapsel und vermeidet eine dorsale Kapselinzision. Beträgt das Gelenkspiel $>20 \mathrm{~mm}$ in a.p. Richtung, muss die dorsale Kapsel gerafft werden. Hierzu legt man entweder dorsal KapsuloraphieNähte oder führt eine in vertikaler Richtung gelegte Kapseldoppelung durch.

\section{Defektarthropathie}

Die Defektarthropathie ist eine der Hauptindikationen zur Implantation einer inversen Schulterprothese. In nur wenigen Fällen stellt die anatomische Schulterprothese eine Alternative zur inversen Prothese dar.

Bei jungen Patienten mit irreversiblen Defekten der Rotatorenmanschette und Gelenkflächenzerstörung, wie sie z.B. nach tiefen Schulterinfekten oder fehlgeschlagener Rotatorenmanschettenrekonstruktion auftreten können, verbietet sich im Hinblick auf die Standzeit des Implantats der Einbau der inversen Schulterprothese. Hier müssen anatomische kraftschlüssige Implantate zur 


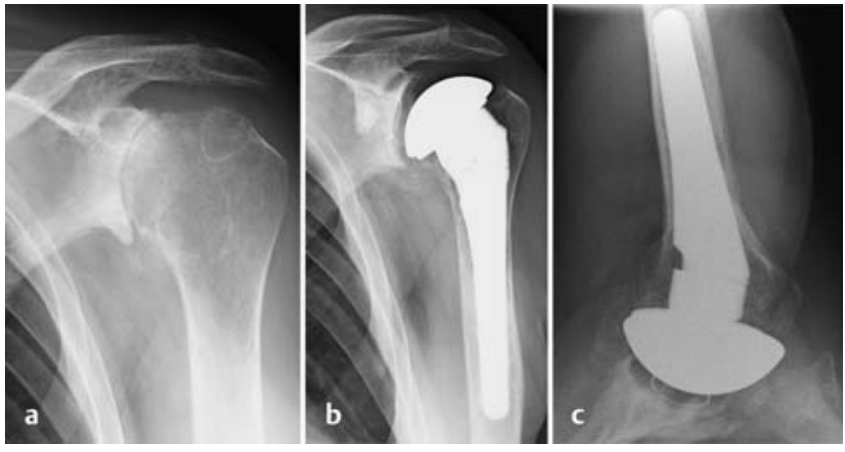

Abb. 11 a bis c a True ap Röntgenbild einer Instabilitätsarthrose Grad III nach Samilson mit medialer Pfannenprotrusion bei Pfannenverbrauch. b True ap Röntgenbild nach Implantation einer Totalprothese der vierten Generation mit zementierter Kiel-Glenoidkomponente. c Axiales Röntgenbild nach Implantation einer Totalprothese der vierten Generation mit zementierter Kiel-Glenoidkomponente.
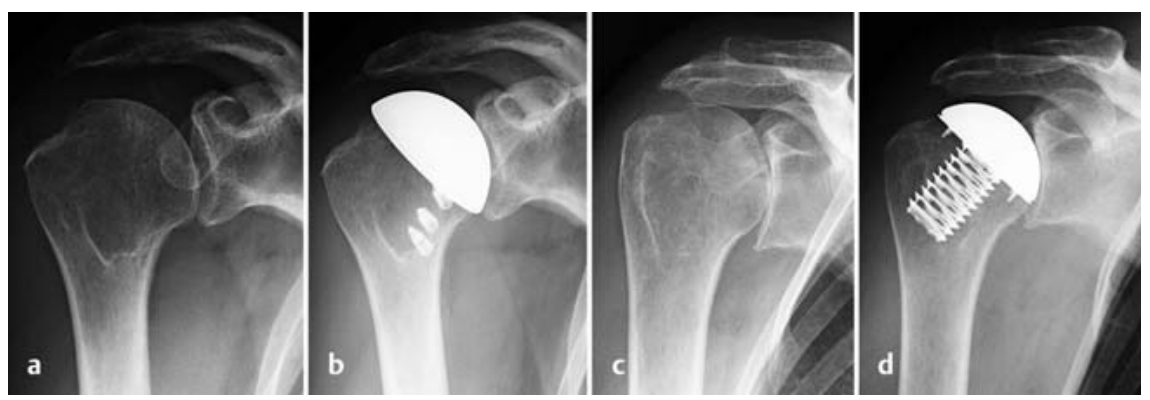

Abb. 12a bis d a True ap Röntgenbild einer Defektarthropathie Grad II nach Hamada. b True ap Röntgenbild nach Cup-Prothese. c True ap Röntgenbild einer Defektarthropathie Grad II nach Hamada. d True ap Röntgenbild nach schaftfreier Prothese.

Anwendung kommen. Hier besteht die Wahl zwischen Humeruskopfersatz mit anatomischer Humeruskopfrekonstruktion und Humeruskopfersatz mit vergrößertem Oberflächen- oder bisphärischem Kopfdesign.

Die Hemiarthroplastik mit anatomischer Humeruskopfrekonstruktion erhält den ehemals physiologischen Roll-Gleit-Mechanismus und vermeidet ein Overstuffing des Glenohumeralgelenks. Die Humeruskopfprothese artikuliert hierbei mit dem erhaltenen korakoakromialen Bogen und dem superioren erodierten Glenoid bei unveränderter Vorspannung des M. deltoideus, was eine akzeptable postoperative Funktion des Glenohumeralgelenks ermöglicht, führt aber nicht zu einem Stabilitätsgewinn [79]. Fast die Hälfte der Patienten erreicht keine völlige Schmerzfreiheit $[80,81]$. Patienten mit einer päoperativen Flexion über $90^{\circ}$ profitieren am meisten von diesem Eingriff. Der Vorteil liegt in der geringeren Komplikationsrate im Vergleich zur inversen Prothese und in der wesentlich besseren Außenrotationsfähigkeit [82].
In Fällen eines gestörten korakoakromialen Bogens oder Akromioplastik nach Voroperationen muss mit schlechteren Resultaten durch anterosuperiore Migration gerechnet werden $[83,84]$.

Neben der 3. und 4. Generation Stielendoprothesen werden hierfür schaftfreie Cup- und Kopfprothesen eingesetzt (Abb. 12), deren Vorteil im problemlosen Wechsel auf inverse Implantate liegt [85].

Unter der Vorstellung, dass der höhergetretene Humeruskopf sowohl mit dem Glenoid als auch mit dem Akromion artikuliert, wurden vergrößerte oder bisphärische Kopfformen gestaltet, die sowohl die glenoidale als auch die akromiale Seite des Humeruskopfs überdecken. Der Gelenkflächenwinkel dieser Prothesen wird dabei vergrößert, ohne dass das laterale Offset oder der Kopfradius geändert werden (CTA Humerusprothese, Fa. DePuy, Lima Ltd; u.a.; Abb. 13).

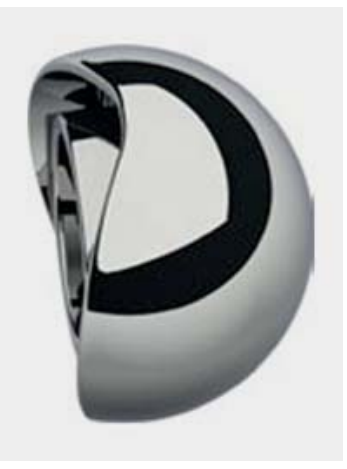

Abb. 13 CTA Humeruskopfprothese (Delta-XTend, Fa. DePuy).

Indikationen für kraftschlüssige Implantate bei der Defektarthropathie

Hierzu eignen sich alle klassischen und analogen Defektarthropathien:

- im Stadium I und II nach Hamada

- im Stadium IA und IIA nach Seebauer (nicht nach medial oder anterior dezentrierte Formen)

- mit vollständigem Erhalt des M. subscapularis

- ohne mediale Pfannenprotrusion

- ohne anterosuperiore Dezentrierung des Humeruskopfs

- bei Patienten, die biologisch jünger als 60 Jahre sind

\section{Kontraindikationen zur anatomischen Schulterendoprothetik}

\section{Absolute Kontraindikationen sind}

- neurogene Arthropathien (Syringomyelie, Charcot-Gelenk),

- irreversible Plexusschädigungen mit Atrophie der Rotatorenmanschette und des M. deltoideus,

- akute und chronische floride Infektionen,

- internistische Erkrankungen.

Der Verlust der sensorischen Gelenkinnervation bei neurologischen Erkrankungen wie der Syringomyelie oder der diabetischen Polyneuropathie kann zur Ausbildung einer Charcot-Arthropathie führen. Durch die fehlende Propriozeption und neurogene Bewegungsstörungen kommt es rasch zur Auslockerung der Prothese mit massiven Osteolysen. Somit ist der prothetische Schultergelenkersatz kontraindiziert. Gleiches gilt für die irreversible Plexusschädigung mit Parese der Rotatorenmanschette und des M. deltoideus. Akute und chronische Schultergelenkinfekte führen ebenfalls zu einer frühzeitigen septischen Prothesenlocke- 
rung. Eine Prothesenimplantation darf daher nur bei sicher nachgewiesenem saniertem Infekt durchgeführt werden.

\section{Relative Kontraindikationen sind}

- fehlende Compliance (z.B. Alkoholismus),

- psychiatrische Erkrankungen.

Als relative Kontraindikationen gelten der unmotivierte, unkooperative Patient, psychiatrische Erkrankungen sowie Alkoholismus, da die postoperative Mitarbeit des Patienten bei der langwierigen, für die postoperative Schultergelenksfunktion wichtigen Rehabilitation nicht gewährleistet ist.

\section{Langzeitergebnisse (Neer-Prothese)}

Deshmukh et al. [86] berichten mit einer Beobachtungszeit zwischen 10 und 23 Jahren über 10-, 15- und 20-JahresÜberlebensraten von 93, 88 und 85\%. Nach mindestens 10 Jahren beurteilten 92\% der Patienten ihre Schulter als besser und viel besser als vor der Implantation bei signifikanter Schmerzreduktion und Funktionsverbesserung.

Die Langzeitanalyse der Neer-TEP weist im Vergleich mit Hüft- und Kniegelenken vergleichbare lange Überlebensraten auf.

Im schwedischen Schulterprothesenregister finden sich zwischen 1999 und 20064283 Meldungen (7\% davon Revisionen). Davon waren 49 Bipolarprothesen und 210 inverse Endoprothesen. Die funktionellen Resultate waren nach HEPgenerell geringer als nach TEP-Implantation (64\% im Western Ontario Osteoarthritis Score vs. 81\%). Erwartete Überlebensraten werden bisher nicht angegeben. Die häufigsten Gründe für Revisionen waren Glenoiderosion, Instabilität, Infektion, Schmerz und Schaftlockerung. Die häufigste Diagnose für Revisionsoperationen waren die Defektarthropathie, Pseudarthrose, Osteonekrose (AVN) und rheumatoide Arthritis (RA). 10,2\% der Bipolarprothesen, 4,5\% der inversen TEP, 2,5\% der HEP und 1,1\% aller implantierten TEP mussten gewechselt werden. Den geringsten Zeitraum von der Indexoperation bis zur Revision hatten die Defektarthropathien, den längsten Patienten mit RA (www.ssas.se/axel).

Tab. 3 Objektive Ergebnisse (Constant Score nach HEP und TEP in Abhängigkeit der Ätiologie [87]).

\begin{tabular}{lll} 
Ätiologie & $\begin{array}{l}\text { Constant Score bei TEP } \\
(\mathbf{n = 2 2 5})\end{array}$ & $\begin{array}{l}\text { Constant Score bei } \\
\text { HEP }(\mathbf{n}=\mathbf{9 9})\end{array}$ \\
\hline primäre Omarthrose & 96,8 & 81 \\
\hline posttraumatische Arthrose & 69 & 65 \\
\hline avaskuläre Nekrose & 82,1 & 82,8 \\
\hline Defektarthropathie & 60 & 65 \\
\hline rheumatoide Arthritis & 79 & 70
\end{tabular}

\section{Ergebnisse in Abhängigkeit der Pathologie}

\section{Omarthrose}

Nach der gegenüberstellenden Analyse von 324 Schulterprothesen [87] weisen die objektiven klinischen Daten in Richtung der totalendoprothetischen Versorgung (siehe Tab.3). Die Unterschiede treten vor allem bei der primären Omarthrose deutlicher hervor. Hier konnten Walch et al. [103] im Vergleich zur HEP signifikant bessere Werte im postoperativen Schmerzempfinden, im Aktivitätslevel wie auch im Gesamt-ConstantScore für die TEP feststellen.

Dies hat sich auch in vielen anderen Nachuntersuchungen so bestätigt [88-94].

\section{Avaskuläre Nekrose}

Die Prothesenimplantation nach atraumatischer avaskulärer Kopfnekrose konnte naturgemäß in den Stadien II bis IV nach Arlet/Ficat bessere Ergebnisse als im Stadium V erzielen [95]. Auch zeigen atraumatische gegenüber posttraumatischen Nekrosen bessere Endergebnisse [96].

Mansat und Mitarbeiter [97] fanden in einer Serie von 19 Patienten mit AVN (6 idiopathisch, 10 kortikosteroidinduziert, 2 Bestrahlungsnekrosen und 1 Patient mit Morbus Gaucher), die in 5 Fällen mit TEP und 14 Fällen mit HEP versorgt wurden, nach 7 Jahren Follow-up 7 exzellente Ergebnisse, 9 befriedigende und 3 unbefriedigende. Der Constant Score lag durchschnittlich bei 58 Punkten (geschlechts- und altersadaptiert 78\%). Lysesäume fanden sich bei 2 Glenoidkomponenten und 1 radiologisch gesicherte Lockerung. In 2 Fällen zeigte sich eine schmerzhafte Glenoiderosion. Insgesamt fand sich also bei $80 \%$ der Patienten eine schmerzlose Schulter mit guten funktionellen Resultaten.
Orfaly et al. [98] fanden bei 21 Patienten (8 posttraumatisch, 1 assoziiert mit Rotatorenmanschettenmassenruptur, 10 kortikosteroidinduziert und 2 idiopathisch) mit einem Durchschnittsalter von 54 Jahren nach 4,7 Jahren bei 15 HEP und 6 TEP eine Verbesserung des ASES-Score auf 81/ 100 und im SST (max. 12 Punkte) auf 10/ 10. Die mittlere aktive Flexion stieg auf $123^{\circ}$ (was verglichen mit $147^{\circ}$ in einer ähnlichen Studie der gleichen Autoren bei Omarthrose deutlich weniger ist [99]), Außenrotation auf $34^{\circ}$ und Innenrotation auf Höhe Th12. Es fand sich kein Unterschied bezüglich Geschlecht oder Alter. Die Patienten hatten präoperativ wesentlich stärkere Schmerzen und Funktionseinschränkungen bei idiopathischer oder kortikosteroidinduzierter AVN, schnitten jedoch postoperativ gleichwertig mit den übrigen Gruppen ab.

Parsch et al. [100] untersuchten 12 Patienten (5 TEP, 8 HEP) mit AVN (exklusive posttraumatische Fälle) nach 30 Monaten und fanden eine Verbesserung des Constant Scores auf 51 (69\% adjustiert), wobei Patienten über 65 deutlich schlechter abschnitten als jüngere. Die Resultate wurden als sehr gut und gut bei 4 von 13 Patienten und als unbefriedigend bei 3 Patienten eingeschätzt, bei denen sich auch Glenoiderosion oder Lysesäume am Glenoid fanden.

\section{Posttraumatische Arthrose/ chronische Fraktur}

In der deutschsprachigen und angloamerikanischen Literatur finden sich bis auf eine prospektive Multicenterstudie nur retrospektive Studien mit inhomogenem Patientengut. Die das funktionelle $\mathrm{Er}$ gebnis beeinflussenden Indikationen zur Prothesenimplantation wie die posttraumatische Arthrose, die posttraumatische Nekrose, Pseudarthrosen und in Fehlstellung verheilte Frakturen werden mit jeweils kleinen Fallzahlen zusammengefasst. 
Insgesamt lässt sich in 62 bis $85 \%$ der Patienten eine deutliche Schmerzreduktion bis hin zur Schmerzfreiheit erzielen.

Die Integrität der Rotatorenmanschette zum Zeitpunkt der Operation hat einen entscheidenden Einfluss auf das funktionelle postoperative Ergebnis. So erzielten Patienten mit intakter Rotatorenmanschette in $77 \%$ der Fälle ein befriedigendes bis exzellentes Ergebnis, wohingegen nur $14 \%$ der Patienten mit Rotatorenmanschettendefekt ein entsprechendes Ergebnis erzielen [101]. Die Autoren untersuchten nach mittleren 47 Monaten 28 Patienten, die in einem durchschnittlichen Alter von 61 Jahren wegen einer posttraumatischen Arthrose (11 Fälle), einer sekundären Nekrose (7 Fälle), einer Pseudarthrose (2 Fälle) oder einer in Fehlstellung verheilten Fraktur (8 Fälle) mit einer Prothese versorgt wurden. In 64,3\% wurden zufrieden stellende bis exzellente Ergebnisse erzielt. Die Flexion verbesserte sich von $71^{\circ}$ präoperativ auf $107^{\circ}$ postoperativ und die Außenrotation stieg von -8 auf $20^{\circ}$ an. Im Constant Score wurden 54 Punkte (alters- und geschlechtsgewichteter Constant Score: $72 \%$ ) erreicht. Norris et al. [120] untersuchten mit einem Mindestbeobachtungszeitraum von 24 Monaten 23 Patienten im mittleren Alter von 53,3 Jahren. Sie führten in $57 \%$ der Fälle eine Osteotomie des Tuberculum majus durch. 61,9\% der Patienten berichteten nur geringe Schmerzen oder waren schmerzfrei. Die Flexion verbesserte sich von $68^{\circ}$ präoperativ auf $92^{\circ}$ postoperativ und die Außenrotation verbesserte sich von $6^{\circ}$ präoperativ auf $27^{\circ}$ postoperativ. Insgesamt sind die Ergebnisse dieser Studie denjenigen der primären Frakturendoprothetik unterlegen. Ursache hierfür kann der hohe Anteil an Tuberculaosteotomien sein, die das postoperative Ergebnis negativ beeinflussen [75]. Anhand einer prospektiven Multicenterstudie mit 71 Patienten, die in einem Durchschnittsalter von 59 Jahren mit einer Prothese versorgt wurden, identifizierten die Autoren 4 verschiedene, das postoperative Ergebnis beeinflussende Frakturtypen. Nach einem mittleren Beobachtungszeitraum von 19 Monaten erzielten $42 \%$ der Patienten ein exzellentes und gutes Ergebnis. Alle Patienten wiesen präoperativ einen Frakturtyp (s. o.) 1 oder 2 auf, die keine Tuberculummajus-Osteotomie erfordern. So wiesen Patienten ohne Tuberculum-majus-Osteotomie eine signifikant bessere Flexion von $123^{\circ}$ auf. Patienten mit Tuberculum-
majus-Osteotomie hingegen erzielten nur eine Flexion von $82^{\circ}$.

Funktionell findet sich bei Typ-1-Frakturen ein Flexionsgewinn von $46^{\circ}\left(87^{\circ}\right.$ präoperativ, $133^{\circ}$ postoperativ) und ein Außenrotationsgewinn von $40^{\circ}\left(0^{\circ}\right.$ präoperativ, $40^{\circ}$ postoperativ). Bei Typ-2Frakturen liegt der Flexionsgewinn bei $57^{\circ}$ (57 ${ }^{\circ}$ präoperativ, $114^{\circ}$ postoperativ) und der Außenrotationsgewinn bei $44^{\circ}$ $\left(-2^{\circ}\right.$ präoperativ, $42^{\circ}$ postoperativ). Bei Typ-3- und -4-Frakturen liegt der Gewinn an Bewegungsausmaß niedriger: Hier wird bei Typ-3-Frakturen ein Flexionsgewinn von $13^{\circ}\left(50^{\circ}\right.$ präoperativ, $63^{\circ}$ postoperativ) und ein Außenrotationsgewinn von $16^{\circ}\left(10^{\circ}\right.$ präoperativ, $26^{\circ}$ postoperativ) beschrieben. Bei Typ-4Frakturen ist der Gewinn an aktivem Bewegungsausmaß etwas besser als bei Typ-3-Frakturen. So beobachten die Autoren einen Gewinn an Flexion von $32^{\circ}$ (58 $8^{\circ}$ präoperativ, $91^{\circ}$ postoperativ) und einen Außenrotationsgewinn von $25^{\circ}$ $\left(0^{\circ}\right.$ präoperativ, $34^{\circ}$ postoperativ). Diese Ergebnisse spiegeln sich auch im Constant Score wieder. Typ-1- und - 2-Frakturen erzielen einen besseren alters- und geschlechtsgewichteten Constant Score mit $73 \%$ als Patienten mit Typ-3- und -4-Frakturen, die einen alters- und geschlechtsgewichteten Constant Score von $58,5 \%$ erreichen.

Eine Voroperation scheint ebenfalls das postoperative Ergebnis negativ zu beeinflussen. So berichten Antuna et al. [102] anhand einer Langzeitbeobachtung von mittleren 9 Jahren, dass voroperierte Patienten eine geringere Schmerzlinderung sowie eine schlechtere Flexion und Außenrotation erzielen als Patienten, die konservativ behandelt wurden (Flexion: 83 vs. $119^{\circ}$; Außenrotation: 21 vs. $40^{\circ}$ ). Auch der negative Einfluss der Tuberculum-majus-Osteotomie wird in dieser Studie bestätigt. Patienten mit Tuberculum-majus-Osteotomie erzielen ebenfalls eine signifikant schlechtere Schulterfunktion (Flexion: 86 vs. $119^{\circ}$; Außenrotation: 27 vs. $44^{\circ}$ ). $58 \%$ der osteotomierten Tubercula heilten in anatomischer Position ein [76].

Schon präoperativ kann die Fehlstellung des Tuberculum majus als negativ Prädiktiver Faktor herangezogen werden [121].

Im eigenen Patientengut wurde neben dem Einfluss der Tuberculum-majusFehlstellung und der Frakturlokalisation (intrakapsulär vs. extrakapsulär) der
Einfluss einer Voroperation auf die Revisionsrate nach chronischer Frakturprothetik beobachtet. So steigt die Revisionsrate durchschnittlich 3,5 Jahre nach chronischer Frakturprothetik bei primär konservativ behandelter Fraktur von 7 auf $17 \%$ bei primär osteosynthetisch behandelten Patienten an.

Die Gesamtkomplikationsrate nach sekundärer Frakturprothesenimplantation liegt bei 27 bis 39\% [75, 122-124].

\section{Rheumatoide Arthritis}

Bei Patienten mit RA konnten Walch et al. [103] mit HEP-Implantation einen alters- und geschlechtskorrigierten Constant Score von 70\% erzielen. Nach Implantation einer Bipolarprothese erzielten Biehl et al. [125] bei intaktem Glenoid und intakter Rotatorenmanschette einen Score von 73. Dagegen wussten Koorevaar et al. [104] nach 8 Jahren Follow-up ein Resultat von nur 37\% zu berichten und beschrieben in über der Hälfte der Fälle ein Fortschreiten der Pfannenerosion.

Patienten mit RA schneiden generell in den meisten Studien nach TEP-Versorgung besser ab als nach HEP, wobei die funktionellen Resultate meist schlechter sind als beim Vorliegen einer primären Omarthrose als Ausgangsdiagnose. Trotzdem zeigen die Patienten eine hohe Zufriedenheit und lange Standzeiten der Implantate $[90,94]$.

In einer prospektiv-randomisierten Studie verglichen Rahme et al. [105] 26 zementierte mit 24 unzementierten Pressfit-Humeruskomponenten bei RA mittels Radiostereometrieanalyse (RSA). In den Nachuntersuchungen bis 2 Jahre nach Implantation hatte sich kein einziger Stiel gelockert, und es fand sich kein Unterschied bezüglich Mikrobewegungen in beiden Gruppen. Die mittlere Rotation betrug $0,25^{\circ}$ und die mittlere Translation 0,32 $\mathrm{mm}$ in beiden Gruppen. Das Einsinken betrug $0,1 \mathrm{~mm}$ bei den unzementierten Schäften. Alle Patienten bis auf 2 waren sehr zufrieden oder zufrieden mit dem Resultat.

Ein wichtiger Faktor für die postoperative Funktion und Zufriedenheit ist der Status der Rotatorenmanschette, die durch die RA in besonderem Maße beeinträchtigt wird. Mengshoel und Slungaard [106] konnten in einer multizentrischen Studie eindeutig zeigen, dass 6 Monate postoperativ die Ergebnisse bei 
intakter Rotatorenmanschette und Bizepssehne signifikant besser waren für die aktive und passive Abduktion und Flexion und die Aktivitäten des täglichen Lebens.

Fink et al. [107] fanden bei 45 Patienten mit RA, die mit einer Durom-Cup-Prothese versorgt wurden, eine Steigerung des Constant Scores auf 66 Punkte bei intakter Rotatorenmanschette und auf 65 bzw. 57 bei kleinen, reparablen bzw. großen irreparablen Rupturen. Alle Patienten waren schmerzfrei, und es gab keine Komplikationen bis zur Nachuntersuchung nach 45 Monaten. In einer jüngsten Studie mit einem Follow-up von 73 Monaten war bei etwa gleichem Constant Score von 64 zwischenzeitlich bei 3 von 35 Patienten eine Revisionsoperation notwendig geworden [108].

In einer prospektiven multizentrischen Studie mit 36 HEP- und 25 TEP-Implantationen fand sich nach 39 Monaten in beiden Gruppen eine signifikante Verbesserung der Funktion und Schmerzsituation. Die TEP-Patienten zeigten dabei eine signifikant bessere aktive Flexion. Der Schweregrad der Erkrankung beeinflusste nicht das klinische Ergebnis. Die Wiederherstellung eines anatomischen Alignements der Endoprothese ging signifikant mit einer Verbesserung der Lebensqualität einher, des Armgebrauchs während der Arbeit und Freizeit und der ROM. Eine Glenoiderosion fand sich bei 4 Patienten mit HEP. Zwei der Glenoidkomponenten waren gelockert [109].

In einer Langzeitstudie von Trail und Nuttall [110] fand sich bei 105 Endoprothesen kein Unterschied zwischen HEP und TEP nach 8,8 Jahren. Eine intakte Rotatorenmanschette war signifikant mit besseren funktionellen Resultaten verknüpft. Keiner der unzementierten Schäfte hatte sich gelockert, 14 Implantate zeigten Lysesäume in einer einzigen Zone. Eine Glenoidkomponente wies radiologisch Lockerungszeichen auf, und 16 hatten Lysesäume in einer Zone, wobei Kielglenoide deutlich schlechter abschnitten. In der HEP-Gruppe fand sich bereits nach 2 Jahren eine superiore Migration des Humerus über $5 \mathrm{~mm}$ bei $28 \%$ und mediale Migration von mehr als $2 \mathrm{~mm}$ bei $16 \%$, wobei sich beides negativ auf das Outcome niederschlug. Vier Patienten wurden revidiert aufgrund der medialen Migration. Mit der Revision als Endpunkt fand sich eine Standzeit von 92\% nach 8 Jahren.

\section{Defektarthropathie}

HEP-Implantationen nach Defektarthropathie wurden in einer neueren Studie [83] in $62,5 \%$ mit und in den restlichen 37,5\% ohne Erfolg durchgeführt. Postel et al. [80] berichten, dass durch HEP-Implantation zwar ein Anstieg des Constant Scores von präoperativ 16 auf postoperativ 34 Punkte erreichbar war, doch gleichzeitig klagte die Hälfte der Patienten weiterhin über Schmerzen. Williams und Rockwood [81] konnten durch eine HEP-Implantation zwar insgesamt den Schmerz deutlich senken, doch auch bei ihnen hatte fast die Hälfte der Patienten weiterhin Schmerzen. In beiden Studien war jedoch eine deutliche Verbesserung der aktiven Flexion festzustellen, bei letzterer sogar von durchschnittlich 70 auf $120^{\circ}$. Arntz et al. [79] berichten nach konventioneller Prothesenimplantation $50 \%$ posteriore Subluxationen.

\section{Instabilitätsarthrose}

Green und Norris [111] untersuchten 19 Patienten, die nach operativer Therapie einer vorderen Instabilität (4 Bristow, 4 Putti-Platt, davon 2 in Kombination mit anderen Operationsverfahren, 4 Magnuson-Stack, 2 Bankart und 5 andere Kapselplastiken) eine sekundäre Arthrose entwickelten. Mit einem Durchschnittsalter von 45 Jahren hatten 15 eine TEP, 2 eine HEP erhalten und $2 \mathrm{~Pa}-$ tienten keine weitere Operation. Die Patienten zeigten eine mittlere Einschränkung der Innenrotationsfähigkeit von $58^{\circ} ; 65 \%$ bedurften einer Subskapularissehnenverlängerung und ausgedehntem Kapsel-Release während der Operation. $18 \%$ benötigten eine Spongiosaplastik und 1 eine dorsale Pfannenaufbauplastik. Nach im Schnitt 62 Monaten zeigte sich eine Schmerzreduktion in $94 \%$, Verbesserung der aktiven Flexion auf $120^{\circ}$, der aktiven Außenrotation auf $41^{\circ}$ und der aktiven Innenrotation auf Höhe Th12. Alle bis auf 1 Patient zeigten eine Verbesserung der Funktion. 12 Schultern wurden subjektiv als viel besser, 4 als besser und 1 als schlechter gegenüber der Situation vor der Operation eingeschätzt. 3 Patienten hatten eine Revisionsoperation.

Matsoukis et al. konnten ein unterschiedliches Muster bezüglich des Alters, des Auftretens der Erstluxation und der Ausbildung einer Arthrose nachweisen. Patienten mit Erstluxation über 40 Jahre und Arthrose waren häufig Frauen mit kurzer Anamnese einer Arthrose und häufigem Vorliegen einer Rotatorenmanschettenruptur. In dieser Gruppe fanden sich gegenüber den häufiger männlichen Patienten mit langer Anamnese ohne Rotatorenmanschettenruptur schlechtere Resultate [112].

Dieselben Autoren untersuchten die Instabilitätsarthrose nicht nur nach operativen Interventionen, sondern auch die Patienten mit chirurgisch unbeeinflusstem „natürlichem“ Verlauf, die dann aufgrund der sekundären Arthrose endoprothetisch versorgt wurden (39TEP, alle Schäfte zementiert). Bei 55 Schultern nach vorderer Luxation (davon 27 mit Stabilisierungsoperation) fand sich nach 45 Monaten eine Verbesserung des Constant Scores von 31 auf 66 Punkte (38 auf $80 \%$ geschlechts- und altersadjustiert). Die aktive Flexion verbesserte sich von 82 auf $139^{\circ}$ und die aktive Außenrotation von 4 auf $39^{\circ}$. 50 Patienten schätzten das Ergebnis als gut oder sehr gut ein. Negative prognostische Faktoren waren das Alter zum Zeitpunkt der Erstluxation und das Vorliegen einer Rotatorenmanschettenläsion. Es fand sich kein Zusammenhang zwischen den demografischen Fakten, der Schulterfunktion vor oder nach Endoprothesenimplantation, den radiologischen Befunden vor oder nach Endoprothesenimplantation, Komplikationsrate und der Reoperationsrate zwischen Patienten mit Voroperation (Stabilisierung) oder „natürlichem“ Verlauf [113].

In einer weiteren Studie untersuchten die gleichen Autoren 27 Patienten mit einem Durchschnittsalter von 56 Jahren nach Schulterendoprothese (21 TEP, 6 HEP), die vorher Stabilisierungsoperationen hatten (zwei Drittel Korakoidtransfer, ein Drittel Weichteiloperation), nach 46 Monaten. Das Durchschnittsalter bei Erstluxation war 32 Jahre mit durchschnittlich 18 Reluxationen. Ein dorsaler Glenoidverbrauch zeigte sich radiologisch bei $24 \%$ der Patienten, eine fettige Degeneration des M. subscapularis bei $45 \%$ und komplette Supraspinatussehnenrupturen in 4 Fällen. Es fanden sich 5 (18\%) Komplikationen inklusive 3 vorderer Instabilitäten (alle über 60 Jahre alt zum Zeitpunkt der 1 . Stabilisierungsoperation), von denen 1 revidiert werden musste. Das Ergebnis war gut oder sehr gut in $56 \%$ der Fälle mit einem normierten Constant Score von 83\%. Die Art der vorhergehenden Stabilisierungsoperation hatte keinen Einfluss auf das postoperative Resultat. Das Vorliegen einer fettigen Degeneration der Rotato- 
renmanschette, insbesondere des $\mathrm{M}$. subscapularis, hatten einen signifikanten Einfluss [114].

Auch Nagels et al. fanden keinen wesentlichen Einfluss der Voroperationen auf das Ergebnis nach endoprothetischer Versorgung [115].

Sperling et al. [116] fanden in einem ähnlichen Patientengut keinen wesentlichen Unterschied zwischen HEP- und TEP-Versorgung bei einem mittleren Follow-up von 2 Jahren und ähnlich gute funktionelle Resultate. Drei Patienten mit HEP und 8 Patienten mit TEP hatten eine Revisionsoperation. Die geschätzten Standzeiten der Endoprothesen war 97\% nach 2 Jahren, 86\% nach 5 Jahren und $61 \%$ nach 10 Jahren.

\section{Literatur}

Boileau P, Walch G. The three-dimensional geometry of the proximal humerus. Implications for surgical technique and prosthetic design. J Bone Joint Surg [Br] 1997; 79: $857-$ 865

2 Iannotti JP, Gabriel JP. Schneck SL et al. The normal glenohumeral relationships. An anatomical study of one hundred and forty shoulders. J Bone Joint Surg [Am] 1992; 74: 491-500

${ }^{3}$ Orr TE, Carter DR. Stress analyses of joint arthroplasty in the proximal humerus. J Orthop Res 1985; 3: 360-371

${ }^{4}$ Rietveld AB, Daanen HA, Rozing PM et al. The lever arm in glenohumeral abduction afte hemiarthroplasty. J Bone Joint Surg $[\mathrm{Br}]$ 1988; 70: 561-565

${ }^{5}$ Boileau P, Walch G. Normal and pathological Anatomy of the Glenoid: Effects on the Design, Preparation and Fixation of the glenoid Component. In: Walch G, Boileau P, editor. Shoulder Arthroplasty. Berlin, Heidelberg: Springer; 1999: 27-140

${ }^{6}$ Neer CS. Rheumatoid Arthritis. In: Neer CS, ed. Shoulder Reconstruction. Philadelphia: WB Saunders; 1990: 212-216

7 Pearl ML, Kurutz S, Robertson DD et al. Geometric analysis of selected press fit prosthetic systems for proximal humeral replacement. J Orthop Res 2002; 20: 192-197

8 Williams jr. GR, Wong KL, Pepe MD et al. The effect of articular malposition after total shoulder arthroplasty on glenohumeral translations, range of motion, and subacromial impingement. J Shoulder Elbow Surg 2001; 10: 399-409

9 Ballmer FT, Sidles JA, Lippitt SB et al. Humeral head prosthetic arthroplasty: Surgically relevant geometric considerations. J Shoulder Elbow Surg 1993; 2: 296-304

${ }^{10}$ Pearl ML, Volk AG. Coronal plane geometry of the proximal humerus relevant to prosthetic arthroplasty. J Shoulder Elbow Surg 1996; 5: 320-326

${ }^{11}$ Jonsson E, Egund N, Kelly I et al. Cup arthroplasty of the rheumatoid shoulder. Acta Orthop Scand 1986: 57: 542-546

12 Copeland SA. Cementless Surface Replacement Arthroplasty for primary Osteoarthritis of the Shoulder. 11 Years Experience. Ann Shoulder Elbow Surg 15th Annual Meeting. New York; 1998
${ }^{13}$ Levy O, Copeland SA. Cementless surface replacement arthroplasty (Copeland CSRA) for osteoarthritis of the shoulder. J Shoulder Elbow Surg 2004; 13: 266-271

14 Neer CS, Brown jr. TH, McLaughlin HL. Fracture of the neck of the humerus with dislocation of the head fragment. Am J Surg 1953; 85: 252-258

${ }^{15}$ Neer 2nd CSI. Glenohumeral Arthroplasty. In: Neer 2nd CSI, ed. Shoulder Reconstruction. Philadelphia: Saunders; 1990: 143-272

${ }^{16}$ Neer 2nd CS. Neer hemiarthroplasty and Neer total shoulder arthroplasty in patients fifty years old or less. Long-term results. J Bone Joint Surg [Am] 1999; 81: 295-296

17 Sperling JW, Cofield RH, Rowland CM. Neer hemiarthroplasty and Neer total shoulder arthroplasty in patients fifty years old or less. Long-term results. J Bone Joint Surg [Am] 1998; 80: 464-473

18 Breusch SJ, Aldinger PR, Thomsen $M$ et al. [Anchoring principles in hip endoprostheses. I: Prosthesis stem]. Unfallchirurg 2000; 103 : 918-931

19 Matsen FA, Rockwood CA, Wirth MA et al. Glenohumeral Arthritis and its Management. In: Rockwood CA, Matsen FA, editor. The Shoulder. Philadelphia: WB Saunders; 1998: 840964

20 Hinrichs F, Boudriot $U$, Griss P. [10 year results with a cemented fine-grit-blasted titaniumaluminum-vanadium hip endoprosthesis shaft]. Z Orthop Ihre Grenzgeb 2000; 138: 52-56

21 Eingartner C, Volkmann R, Winter E et al. Results of a cemented titanium alloy straight femoral shaft prosthesis after 10 years of follow-up. Int Orthop 2001; 25: 81-84

22 Sedel L, Nizard RS, Kerboull L et al. Aluminaalumina hip replacement in patients younger than 50 years old. Clin Orthop Relat Res 1994: 175-183

${ }^{23}$ Neuss M, Clemens S, Marx R et al. [Is the implantation of titanium stems in hip prostheses really obsolete?]. Z Orthop Ihre Grenzgeb 2005; 143: 337-342

24 Crowninshield RD, Brand RA, Johnston RC et al. The effect of femoral stem cross-sectional geometry on cement stresses in total hip reconstruction. Clin Orthop Relat Res 1980: 71-77

25 Cofield RH. Uncemented total shoulder arthroplasty. A review. Clin Orthop Relat Res 1994: 86-93

${ }^{26}$ Robertson DD, Yuan J, Bigliani LU et al. Threedimensional analysis of the proximal part of the humerus: relevance to arthroplasty. J Bone Joint Surg [Am] 2000; 82: 1594-1602

27 Matsen 3rd FA, Iannotti JP, Rockwood jr. CA. Humeral fixation by press-fitting of a tapered metaphyseal stem: a prospective radiographic study. J Bone Joint Surg [Am] 2003; 85: 304-308

28 Peppers TA, Jobe CM, Dai QG et al. Fixation of humeral prostheses and axial micromotion. J Shoulder Elbow Surg 1998; 7: 414-418

${ }^{29}$ Nyffeler RW, Sheikh R, Jacob HA et al. Influence of humeral prosthesis height on biomechanics of glenohumeral abduction. An in vitro study. J Bone Joint Surg [Am] 2004; 86: 575-580

30 Poppen NK, Walker PS. Forces at the glenohumeral joint in abduction. Clin Orthop Relat Res 1978: 165-170

${ }^{31}$ Habermeyer P, Schweiberer L. [Corrective interventions subsequent to humeral head fractures]. Orthopade 1992; 21: 148-157

32 Boileau P, Walch G, Liotard JP. [Radio-cinematographic study of active elevation of the prosthetic shoulder]. Rev Chir Orthop Reparatrice Appar Mot 1992; 78: 355-364
33 Barrett WP, Franklin JL, Jackins SE et al. Total shoulder arthroplasty. J Bone Joint Surg [Am] 1987; 69: 865-872

${ }^{34}$ Swieszkowski W, Bednarz P, Prendergast PJ. Contact stresses in the glenoid component in total shoulder arthroplasty. Proc Inst Mech Eng H 2003; 217: 49-57

${ }^{35}$ Flatow EL. Prosthetic design considerations in total shoulder arthroplasty. Semin Arthroplasty 1995; 6: 233-244

36 Harryman DT, Sidles JA, Harris SL et al. The effect of articular conformity and the size of the humeral head component on laxity and motion after glenohumeral arthroplasty. A study in cadavera. J Bone Joint Surg [Am] 1995; 77: 555-563

${ }^{37}$ Friedman RJ. Biomechanics and Design of Shoulder Arthroplasties. In: Friedman RJ, editor. Arthroplasty of the Shoulder. Stuttgart, New York: Thieme; 1994: 27-40

38 Terrier A, Buchler P, Farron A. Influence of glenohumeral conformity on glenoid stresses after total shoulder arthroplasty. J Shoulder Elbow Surg 2006; 15: 515-520

${ }^{39}$ Anglin C, Wyss UP, Nyffeler RWet al. Loosening performance of cemented glenoid prosthesis design pairs. Clin Biomech (Bristol, Avon) 2001; 16: 144-150

40 Walch G, Edwards TB, Boulahia A et al. The influence of glenohumeral prosthetic mismatch on glenoid radiolucent lines: results of a multicenter study. J Bone Joint Surg [Am] 2002; 84: 2186-2191

${ }^{41}$ Diop A, Maurel N, Grimberg J et al. Influence of glenohumeral mismatch on bone strains and implant displacements in implanted glenoids. An in vitro experimental study on cadaveric scapulae. J Biomech 2006; 39: 3026 3035

42 Nho SJ, Ala OL, Dodson CC et al. Comparison of conforming and nonconforming retrieved glenoid components. J Shoulder Elbow Surg 2008; 17: 914-920

${ }^{43}$ Hertel R, Ballmer FT. Observations on retrieved glenoid components. J Arthroplasty 2003; 18: 361-366

${ }^{44}$ Collins D, Tencer A, Sidles J et al. Edge displacement and deformation of glenoid components in response to eccentric loading. The effect of preparation of the glenoid bone. J Bone Joint Surg [Am] 1992; 74: 501-507

45 Szabo I, Buscayret F, Edwards TB et al. Radiographic comparison of flat-back and convexback glenoid components in total shoulder arthroplasty. J Shoulder Elbow Surg 2005; 14: 636-642

${ }^{46}$ Nyffeler RW, Sheikh R, Jacob H et al. The Relevance of Orientation of the glenoid Component in total Shoulder Arthroplasty. 8th ICSS. Cape Town, South Africa; 2001

47 Spencer jr. EE, Valdevit A, Kambic $\mathrm{H}$ et al. The effect of humeral component anteversion on shoulder stability with glenoid component retroversion. J Bone Joint Surg [Am] 2005; 87: 808-814

48 Iannotti JP, Spencer EE, Winter $U$ et al. Prosthetic positioning in total shoulder arthroplasty. J Shoulder Elbow Surg 2005; 14 (Suppl. 1): 111S-121S

49 Hertel $R$, Lehmann 0 . [Glenohumeral joint. Anatomical aspects and implications for prosthesis design]. Orthopade 2001; 30: 363-369

50 Oosterom R. Herder JL, van der Helm FC et al. Translational stiffness of the replaced shoulder joint. J Biomech 2003; 36: 1897-1907

51 Friedman RJ, Laberge M, Dooley RL et al. Finite element modeling of the glenoid component: Effect of design parameters on stress distribution. J Shoulder Elbow Surg 1992; 1: 261270 
52 Murphy LA, Prendergast PJ, Resch H. Structural analysis of an offset-keel design glenoid component compared with a center-keel design. J Shoulder Elbow Surg 2001; 10: 568579

53 Nyffeler RW, Meyer D, Sheikh R et al. The effect of cementing technique on structural fixation of pegged glenoid components in total shoulder arthroplasty. J Shoulder Elbow Surg 2006; 15: 106-111

${ }^{54}$ Wirth MA, Rockwood jr. CA. Complications of total shoulder-replacement arthroplasty. J Bone Joint Surg [Am] 1996; 78: 603-616

55 Giori NJ, Beaupre GS, Carter DR. The influence of fixation peg design on the shear stability of prosthetic implants. J Orthop Res 1990; 8: 892-898

56 Jensen $K L$. The effects of selected classical music on self-disclosure. J Music Ther 2001; 38 . $2-27$

57 Lazarus MD, Jensen KL, Southworth C et al. The radiographic evaluation of keeled and pegged glenoid component insertion. J Bone Joint Surg [Am] 2002; 84: 1174-1182

58 Gartsman GM, Elkousy HA, Warnock KM et al. Radiographic comparison of pegged and keeled glenoid components. J Shoulder Elbow Surg 2005; 14: 252-257

59 Stone KD, Grabowski JJ, Cofield RH et al. Stress analyses of glenoid components in total shoulder arthroplasty. J Shoulder Elbow Surg 1999; 8: 151-158

60 Barwood S, Setter KJ, Blaine TA et al. The incidence of early radiolucencies about a pegged glenoid component using cement pressurization. J Shoulder Elbow Surg 2008; 17: 703708

61 Nyffeler RW, Sheikh R, Atkinson TS et al. Effects of glenoid component version on humeral head displacement and joint reaction forces: an experimental study. J Shoulder Elbow Surg 2006; 15: 625-629

62 Nyffeler RW, Anglin C, Sheikh R et al. Influence of peg design and cement mantle thickness on pull-out strength of glenoid component pegs. J Bone Joint Surg [Br] 2003; 85: 748752

63 Andreykiv A, Prendergast PJ, van Keulen F et al. Bone ingrowth simulation for a concept glenoid component design. J Biomech 2005; 38 : 1023-1033

64 Gazielly DF. Controversies in Shoulder Arthroplasty. Paris; 2005

65 Habermeyer P, Magosch P, Lichtenberg S. Recentering the humeral head for glenoid deficiency in total shoulder arthroplasty. Clin Orthop Relat Res 2007; 457: 124-132

66 Pelletier MH, Langdown A, Gillies RM et al. Photoelastic comparison of strains in the underlying glenoid with metal-backed and all-polyethylene implants. J Shoulder Elbow Surg 2008; 17: 779-783

67 Mullett H, Levy O, Raj D et al. Copeland surface replacement of the shoulder. Results of an hydroxyapatite-coated cementless implant in patients over 80 years of age. J Bone Joint Surg [Br] 2007; 89: 1466-1469

68 Smith KL, Matsen 3rd FA. Total shoulder arthroplasty versus hemiarthroplasty. Current trends. Orthop Clin North [Am] 1998; 29: 491-506

69 Cofield RH, Frankle MA, Zuckerman JD. Humeral head replacement for glenohumeral arthritis. Semin Arthroplasty 1995; 6: 214-221

70 Levine WN, Djurasovic M, Glasson JM et al. Hemiarthroplasty for glenohumeral osteoarthritis: results correlated to degree of glenoid wear. J Shoulder Elbow Surg 1997; 6: 449454

71 Kempf JF, Gleyze P, Bonnomet F et al. A multicenter study of 210 rotator cuff tears treated by arthroscopic acromioplasty. Arthroscopy 1999; 15: 56-66

72 Boyd jr. AD, Thomas WH, Scott RD et al. Total shoulder arthroplasty versus hemiarthroplasty. Indications for glenoid resurfacing. J Arthroplasty 1990; 5: 329-336

73 Boileau P, Walch G, Trojani C et al. Sequelae of Fractures of the proximal Humerus: Results of Shoulder Arthroplasty without greater Tuberosity Osteotomy. In: Walch G, Boileau P, editor. Shoulder Arthroplasty. Berlin, Heidelberg, New York: Springer; 1998: 359-369

74 Boileau P, Chuinard C, Le Huec JC et al. Proximal humerus fracture sequelae: impact of a new radiographic classification on arthroplasty. Clin Orthop Relat Res 2006; 442: 121-130

75 Boileau P, Trojani C, Walch Get al. Shoulder arthroplasty for the treatment of the sequelae of fractures of the proximal humerus. I Shoulder Elbow Surg 2001; 10: 299-308

76 Mansat P, Guity MR, Bellumore Y et al. Shoulder arthroplasty for late sequelae of proximal humeral fractures. J Shoulder Elbow Surg 2004; 13: 305-312

77 Thomas T, Noel E, Goupille P et al. The rheumatoid shoulder: current consensus on diagnosis and treatment. Joint Bone Spine 2006; 73: $139-143$

78 Schill S, Thabe H, Grifka J. [Differential therapy for the rheumatoid shoulder]. Orthopade 2002; 31: 1132-1144

${ }^{79}$ Arntz CT, Jackins S, Matsen 3rd FA. Prosthetic replacement of the shoulder for the treatment of defects in the rotator cuff and the surface of the glenohumeral joint. J Bone Joint Surg [Am] 1993; 75: 485-491

80 Postel JM, Allain J, Favard L et al. Osteoarthritis with massive Cuff Tear: Results of an unconstrained Shoulder Arthroplasty. In: Walch G, Boileau P, eds. Shoulder Arthroplasty. Berlin, Heidelberg, New York: Springer; 1999: 271-275

81 Williams jr. GR, Rockwood jr. CA. Hemiarthroplasty in rotator cuff-deficient shoulders. J Shoulder Elbow Surg 1996; 5: 362-367

82 Goldberg SS, Bell JE, Kim HJ et al. Hemiarthroplasty for the rotator cuff-deficient shoulder. J Bone Joint Surg [Am] 2008; 90: 554-559

83 Field LD, Dines DM, Zabinski SJ et al. Hemiarthroplasty of the shoulder for rotator cuff arthropathy. J Shoulder Elbow Surg 1997; 6: 18-23

84 Sanchez-Sotelo J, Cofield RH, Rowland CM. Shoulder hemiarthroplasty for glenohumeral arthritis associated with severe rotator cuff deficiency. J Bone Joint Surg [Am] 2001; 83: 1814-1822

85 Jerosch J, Schunck J, Morsy MG. [Shoulder resurfacing in patients with rotator cuff arthropathy and remaining subscapularis function]. Z Orthop Unfall 2008; 146: 206-210

${ }^{86}$ Deshmukh AV, Koris M, Zurakowski D et al. Total shoulder arthroplasty: long-term survivorship, functional outcome, and quality of life. J Shoulder Elbow Surg 2005; 14: 471479

87 Walch G, Boulahia A, Badet $R$ et al. Primary glenohumeral Osteoarthritis: Clinical and radiographic Classification. In: Walch G, Boileau P, editor. Shoulder Arthroplasty. Berlin, Heidelberg: Springer; 1999: 195-201

88 Adams JE, Sperling JW, Hoskin TL et al. Shoulder arthroplasty in Olmsted County, Minnesota, 1976-2000: a population-based study. J Shoulder Elbow Surg 2006; 15: 50-55

89 Adams JE, Sperling JW, Schleck CD et al. Outcomes of shoulder arthroplasty in Olmsted County, Minnesota: a population-based study. Clin Orthop Relat Res 2007; 455: 176182
90 Bryant D, Litchfield R, Sandow M et al. A comparison of pain, strength, range of motion, and functional outcomes after hemiarthroplasty and total shoulder arthroplasty in patients with osteoarthritis of the shoulder. A systematic review and meta-analysis. J Bone Joint Surg [Am] 2005; 87: 1947-1956

91 Haines JF, Trail IA, Nuttall D et al. The results of arthroplasty in osteoarthritis of the shoulder. J Bone Joint Surg [Br] 2006; 88: 496-501

92 Iannotti JP, Norris TR. Influence of preoperative factors on outcome of shoulder arthroplasty for glenohumeral osteoarthritis. J Bone Joint Surg [Am] 2003; 85: 251-258

93 Tang KL, Li QH, Habermeyer P. [Clinical outcomes of treatment of glenohumeral osteoarthritis with new generation shoulder prosthesis]. Zhonghua Yi Xue Za Zhi 2006; 86: 2741-2748

94 van de Sande MA, Brand R, Rozing PM. Indications, complications, and results of shoulder arthroplasty. Scand J Rheumatol 2006; 35: 426-434

95 Basso M, Nové-Josserand L. Results of the Arthroplasty for atraumatic avascular Necrosis of the humeral Head. In: Walch G, Boileau P, editor. Shoulder Arthroplasty. Berlin, Heidelberg: Springer; 1999: 251-257

96 Hattrup SJ. Indications, technique, and results of shoulder arthroplasty in osteonecrosis. Orthop Clin North Am 1998; 29: 445-451

97 Mansat $P$, Huser L, Mansat $M$ et al. Shoulder arthroplasty for atraumatic avascular necrosis of the humeral head: nineteen shoulders followed up for a mean of seven years. J Shoulder Elbow Surg 2005; 14: 114-120

98 Orfaly RM, Rockwood jr. CA, Esenyel CZ et al. Shoulder arthroplasty in cases with avascular necrosis of the humeral head. J Shoulder Elbow Surg 2007; 16 (Suppl. 3): S27-S32

99 Orfaly RM, Rockwood jr. CA, Esenyel CZ et al. A prospective functional outcome study of shoulder arthroplasty for osteoarthritis with an intact rotator cuff. J Shoulder Elbow Surg 2003; 12: 214-221

100 Parsch D, Lehner B, Loew M. Shoulder arthroplasty in nontraumatic osteonecrosis of the humeral head. J Shoulder Elbow Surg 2003; 12: 226-230

101 Kralinger F, Schwaiger R, Wambacher M et al. Outcome after primary hemiarthroplasty for fracture of the head of the humerus. A retrospective multicentre study of 167 patients. J Bone Joint Surg [Br] 2004; 86: 217219

102 Antuna SA, Sperling JW, Sanchez-Sotelo J et al. Shoulder arthroplasty for proximal humeral malunions: long-term results. J Shoulder Elbow Surg 2002; 11: 122-129

103 Walch G, Boileau P, Pozzi I. Glenoid resurfacing in Shoulder Arthroplasty: pro's and con's. In: Walch G, Boileau P, editor. Shoulder Arthroplasty. Berlin, Heidelberg: Springer; 1999: 177-181

104 Koorevaar RC, Merkies ND, de Waal Malefijt $M C$ et al. Shoulder hemiarthroplasty in rheumatoid arthritis. 19 cases reexamined after 1-17 years. Acta Orthop Scand 1997; 68: 243-245

105 Rahme H, Mattsson P, Wikblad L et al. Cement and press-fit humeral stem fixation provides similar results in rheumatoid patients. Clin Orthop Relat Res 2006; 448: 2832

106 Mengshoel AM, Slungaard B. Effects of shoulder arthroplasty and exercise in patients with rheumatoid arthritis. Clin Rheumatol 2005; 24: 258-265 
107 Fink B, Singer J, Lamla $U$ et al. Surface replacement of the humeral head in rheumatoid arthritis. Arch Orthop Trauma Surg 2004; 124: 366-373

108 Fuerst M, Fink B, Ruther $W$. The DUROM cup humeral surface replacement in patients with rheumatoid arthritis. Surgical technique. J Bone Joint Surg [Am] 2008; 90 (Suppl. 2): 287-298

109 Collins DN, Harryman 2nd DT, Wirth MA Shoulder arthroplasty for the treatment of inflammatory arthritis. J Bone Joint Surg [Am] 2004; 86: 2489-2496

110 Trail IA, Nuttall D. The results of shoulder arthroplasty in patients with rheumatoid arthritis. J Bone Joint Surg [Br] 2002; 84 1121-1125

111 Green A, Norris TR. Shoulder arthroplasty for advanced glenohumeral arthritis after anterior instability repair. J Shoulder Elbow Surg 2001; 10: 539-545

112 Matsoukis J, Tabib W, Mandelbaum A et al. [Shoulder arthroplasty for non-operated anterior shoulder instability with secondary osteoarthritis]. Rev Chir Orthop Reparatrice Appar Mot 2003; 89: 7-18

113 Matsoukis J, Tabib W, Guiffault P et al. Shoulder arthroplasty in patients with a prior anterior shoulder dislocation. Results of a multicenter study. J Bone Joint Surg [Am] 2003 85: 1417-1424

114 Matsoukis J, Tabib W, Guiffault $P$ et al. [Shoulder arthroplasty for osteoarthritis after prior surgery for anterior instability: a report of 27 cases]. Rev Chir Orthop Reparatrice Appar Mot 2003; 89: 580-592
115 Nagels J, Valstar ER, Stokdijk M et al. Patterns of loosening of the glenoid component. J Bone Joint Surg [Br] 2002; 84: 83-87

116 Sperling JW, Antuna SA, Sanchez-Sotelo J et al. Shoulder arthroplasty for arthritis after instability surgery. J Bone Joint Surg [Am] 2002; 84: 1775-1781

117 Staffee AD, More RW. Hemi-resurfacing arthroplasty of the rheumatoid shoulder. Contemp Orthop 1984; 9: 51-59

118 Cruess RL. Osteonecrosis of bone. Current concepts as to etiology and pathogenesis. Clin Orthop Relat Res 1986; 208: 30-39

119 Moineau G, McClelland WB, Troijani $C$ et al. Prognostic factors and limitations of anatomic shoulder arthroplasty for the treatment of posttraumatic cephalic collapse or necrosis. J Bone Joint Surg [Am] 2012; 94: 2186-2194

120 Norris TR, Green A, Mc Guigan FX. Late prosthetic shoulder arthroplasty for displaced proximal humerus fractures. J Shoulder Elbow Surg 1995; 4: 271-280

121 Tauber M, Karpik S, Matis $N$ et al. Shoulder arthroplasty for traumatic avascular necrosis: predictors of outcome. Clin Orthop Relat Res 2007, 465: 208-214

122 Beredjiklian PK, Iannotti JP. Treatment of proximal humerus fracture malunion with prosthetic arthroplasty. Instr Course Lect 1998; 47: 135-140

123 Boileau P, Watkinson D, Hatzidakis AM et al. Neer Award 2005: The Grammont reverse shoulder prosthesis: results in cuff tear arthritis, fracture sequelae, and revision arthroplasty. J Shoulder Elbow Surg 2006; 15: 527-540
124 Habermeyer P, Lichtenberg S, Magosch P. Sind die Ergebnisse der sekundären SchulterFrakturprothetik abhängig vom Frakturtyp nach Boileau? Deutscher Kongress für Orthopädie und Unfallchirurgie 2008, 22.25.10.2008; Berlin

125 Biehl C, Schill J, Thabe H. Die rekonstruktive Versorgung der Rheumatischen Schulter mit der MVS-Schulterprothese. 47. Jahrestagung der NOV, Leipzig; 1998

\section{Dr. med. Petra Magosch}

Facharzt für Orthopädie und Unfallchirurgie. Sportmedizin, Medizinische Informatik

Schulter- und Ellenbogenchirurgie

Dr. med. Sven Lichtenberg

Facharzt für Orthopädie und Unfallchriurgie, Sportmedizin

Schulter- und Ellenbogenchirurgie

Prof. Dr. med. Peter Habermeyer Facharzt für Chirurgie, Orthopädie und Unfallchirurgie, Sportmedizin Schulter- und Ellenbogenchirurgie

ATOS Klinik

Bismarckstraße 9-15

69115 Heidelberg

petra.magosch@atos.de 